\title{
The Random Trip Model: Stability, Stationary Regime, and Perfect Simulation ${ }^{12}$
}

\author{
March 2006 \\ Technical Report \\ MSR-TR-2006-26
}

Jean-Yves Le Boudec and Milan Vojnović

\author{
Microsoft Research \\ Microsoft Corporation \\ One Microsoft Way \\ Redmond, WA 98052 \\ http://www.research.microsoft.com
}

\footnotetext{
${ }^{1}$ The conference version of the paper appeared at IEEE INFOCOM 2005, Miami, FL, March 2005, under the title "Perfect Simulation and Stationarity of a Class of Mobility Models".

${ }^{2}$ Author affiliations: Jean-Yves Le Boudec, EPFL, CH-1015, Lausanne, Switzerland, Email: jean-yves.leboudec@epfl.ch; Milan Vojnović, Microsoft Research Ltd, CB3 0FB Cambridge, United Kingdom, Email: milanv@ microsoft.com.
} 
Abstract- We define "random trip", a generic mobility model for random, independent node motions, which contains as special cases: the random waypoint on convex or non convex domains, random walk on torus, billiards, city section, space graph, intercity and other models. We show that, for this model, a necessary and sufficient condition for a timestationary regime to exist is that the mean trip duration (sampled at trip endpoints) is finite. When this holds, we show that the distribution of node mobility state converges to the time-stationary distribution, starting from origin of an arbitrary trip. For the special case of random waypoint, we provide for the first time a proof and a sufficient and necessary condition of the existence of a stationary regime, thus closing a long standing issue. We show that random walk on torus and billiards belong to the random trip class of models, and establish that the time-limit distribution of node location for these two models is uniform, for any initial distribution, even in cases where the speed vector does not have circular symmetry. Using Palm calculus, we establish properties of time-stationary regime, when the condition for its existence holds. We provide an algorithm to sample the simulation state from a time-stationary distribution at time 0 ("perfect simulation"), without computing geometric constants. For random waypoint on the sphere, random walk on torus and billiards, we show that, in the time-stationary regime, the node location is uniform. Our perfect sampling algorithm is implemented to use with ns-2, and is available to download from http://ica1www.epfl.ch/RandomTrip.

\section{INTRODUCTION}

$\mathbf{R}$ ANDOM mobility models have been used extensively to evaluate performance of networking systems in both mathematical analysis and simulation based studies. The goal of our work is twofold: (i) provide a class of "stable" mobility models that is rich enough to accommodate a large variety of examples and (ii) provide an algorithm to run "perfect simulation" of these models. Both goals are motivated by recent findings about the random waypoint; this is an apparently simple model that fits in our framework, the simulation of which was reported to pose a surprising number of challenges, such as speed decay, a change in the distribution of location and speed as the simulation progresses [26], [18], [24], [12].

\section{A. Random Trip Model}

We define "random trip", a model of random, independent node movements. Such independent node movements are entirely defined by specifying random process of movement for a single node. The model does not directly accommodate group mobility models, which are left for further study. The random trip model is defined by a set of "stability" conditions for a node movement. These conditions guarantee existence of a time-stationary regime of node mobility state or its non existence. They also guarantee convergence of node mobility state to a time-stationary regime, whenever one exists, starting a node movement from origin of a trip. The reported observations for random waypoint such as that speed vanishes to 0 as simulation progresses ("considered harmful" [25]) are in fact all related to the set of problems on stability of random processes that include finding conditions for existence of a stationary regime or its non existence. Stability problems also include finding conditions under which convergence to a stationary regime is guaranteed, whenever there exists one. These conditions are important to alleviate non desirable situations such as the reported vanishing of node numerical speed to 0 .

In the absence of established properties of real mobility patterns, it is not yet clear today what the requirements on mobility models should be [10]. The random trip model is a broad class of independent node movements that can be appropriately parameterised to synthesise an a priori assumed mobile behaviour.

\section{B. Random Trip Examples}

We show in Section III that many examples of random mobility models used in practise are random trip models. Our catalogue includes examples such as classical random waypoint, city driving models ("space graph" [14], "city section" or "hierarchical random waypoint" [6]), circulation models ("random waypoint on sphere"), or the special purpose "fish in a bowl" and "Swiss flag". These are all accommodated by the "restricted random waypoint" introduced in Section IIID. These examples illustrate well the geometric diversity of mobility domains: for models such as "Swiss flag" we have a non convex area on a plane; for models such as "space graph" or "city-section", a concatenation of line segments that represent streets; for "random waypoint on sphere", a surface in a three dimensional space.

In some cases, it is desirable to assume that in steadystate, node location is uniformly distributed on a domain. This is provided by "random walk on torus" and "billiards", which are defined by "bending" the paths of node movement with wrapping and billiards-like reflections, respectively, in a rectangular area on a plane. "Random waypoint on a sphere" is another such example, embedded in three dimensions.

\section{Perfect Simulation}

Like many simulation models, when the condition for stability is satisfied, simulation runs go through a transient period and converge to the stationary regime. It is important to remove the transients for performing meaningful comparisons of, for example, different mobility regimes. A standard method for avoiding such a bias is to (i) make sure the used model has a stationary regime and (ii) remove the beginning of all simulation runs in the hope that long runs converge to stationary regime.

However, as we show now, the length of transients may be prohibitively long for even simple mobility models. Our example is the space graph explained in Figure 2. There are a little less than 5000 possible paths; in Figure 2 we show the distribution of the path used by the mobile at time $t$, given that initially a path is selected uniformly among all possible paths (i.e. the mobile is initially placed at a random vertex (uniformly) and the trip destination vertex is also drawn uniformly at random on the set of the vertices). This was 


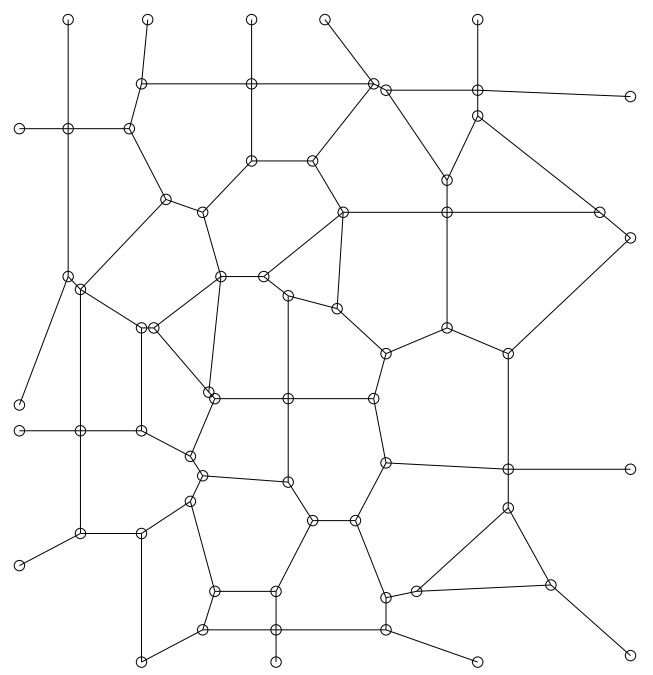

Fig. 1. Mobility on a "Space Graph" as introduced by Jardosh et al [14]. A mobile initiates a trip from a vertex and moves along a shortest-path to a randomly chosen destination vertex. This model is discussed in Section IIID.2. The alternative, called city section (Section III-C.2), chooses the trip end-points on any point of the domain defined by the line segments of the graph edges. The spatial graph is either generated synthetically (e.g. [14] or constructed from real-world street maps. The numeric speeds can be assigned to the edges of a graph.

obtained analytically (details are in Appendix M). Figure 2 illustrates that the transient period may be long compared to typical simulation lengths (for example $900 \mathrm{sec}$ in [9]).

A major difficulty with transient removal is to know when the transient ends; if it may be long, as we illustrated, considerable care should be used. An alternative, called "perfect simulation", is to sample the initial simulation state from the stationary regime. For most models this is hard to do, but, as we show, this is quite easy (from an implementation viewpoint) for the random trip model. Perfect simulation for the random waypoint was advocated and solved by Navidi and Camp in [22] who also give the stationary distribution (assuming location and speed are independent in the stationary regime, an issue later resolved in [16] using the Palm techniques in this paper).

\section{The Palm Calculus Framework}

The derivations in [22] involve long and sophisticated computations. We use a different approach, based on Palm calculus, a set of formulae that relate time averages to event averages. Palm calculus is now well established, but not widely used or even known in applied areas. For a quick overview of Palm calculus, see [17]; for a full fledged theory, see [3]. This framework allows us to generalise the results in [22] to the broad class of restricted random waypoint models, and obtain a sampling algorithm that, for complicated, non convex areas, does not require a priori computation of geometric integrals. More fundamentally, the Palm calculus framework allows us derive simple sampling algorithms for the generic random trip model — a task that may be formidable without this tool.
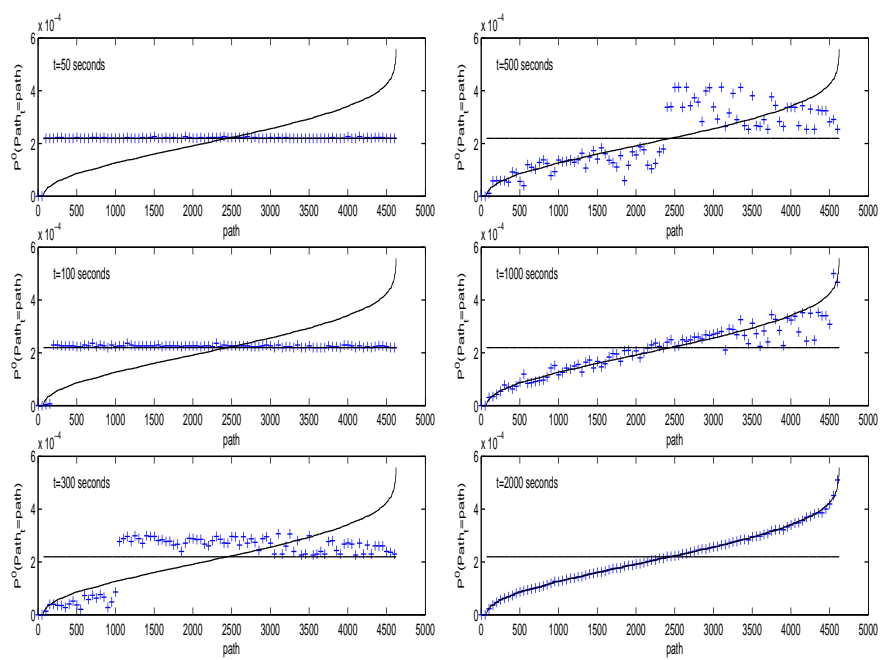

Fig. 2. The probability distribution of the path at time $t$ for a mobile on the space graph in Figure 1. The node numerical speed is fixed to $1.25 \mathrm{~m} / \mathrm{s}$. The space graph spans a $1 \mathrm{~km} \times 1 \mathrm{~km}$ area. The initial path is chosen uniformly among all possible paths. $x$-axis: path index, ordered by path length; $y$-axis: probability that this path is used at time $t$ for $t=50,100,300,500,1000,2000$ seconds of simulated time. Horizontal solid line: initial distribution; other solid line: the time-stationary distribution. The transient lasts for a long time.

\section{E. Summary of Main Contributions}

Our main contributions are summarised as follows:

- We provide "random trip model", a generic mobility model with a framework for analysis.

- We identify a necessary and sufficient condition for existence of a time stationary regime for random trip model. This appears to be a new result even for the classical random waypoint, and fully explains the reported "harmfulness" [25].

- We show that random trip models feature convergence in distribution of node mobility state to a time-stationary regime, from origin of an arbitrary trip.

- In particular, we prove that a node location for "random walk on torus" and "billiards" at trip transition instants converges to the uniform distribution on a rectangular area, from any initial distribution. For the "random walk on torus" model, the result requires a mild assumption on the distribution of the node speed vector (essentially, that it has a density) whereas previous results in [21] required the circular symmetry (speed vector is isotropic). For the "billiards" model, we require that the speed vector has a completely symmetric distribution (Section III-G), which means that it goes up or down [resp. left or right] with equal probability. This is also a weaker assumption than the circular symmetry required in [21].

- We show that for three examples (random walk on torus, billiards, random waypoint on sphere) the node location is uniform in steady-state. For the random walk on torus, the steady state is essentially the same as the naive initialisation (with uniform node placement) and there is no speed decay. In contrast, there is speed decay for random waypoint on a sphere.

- We provide an algorithm to initialise node mobility state so that the distribution of the node state is time-stationary throughout a simulation ("perfect simulation").

- The perfect sampling algorithm (i) accommodates ran- 
dom waypoint models on non convex areas and (ii) avoids computation of geometric integrals when they are difficult to compute.

\section{F. Organisation of the Paper}

The random trip model is defined in Section II, along with a notation list. Section III provides a broad catalogue of random mobility models and shows that all are random trip models. In particular, that section contains convergence results for "random walk on torus" and "billiards" random trip models. The main result on stability is the necessary and sufficient condition for existence and uniqueness of a timestationary regime, and convergence to this regime, whenever it exists, is given in Section IV. In Section V we give a generic representation of the time stationary distribution of any random trip model that satisfies the stability condition. In Section VI we derive an efficient sampling algorithm for perfect simulation for the sub-family of models that can be represented as restricted random waypoint. In Section VII we show that, for random waypoint on sphere, random walk on torus and billiards, the time-stationary distribution of node location is uniform, i.e. the distribution bias for location does not exist for these models. In Section VIII we discuss related work. Section IX provides concluding remarks. Most of the proofs are deferred to the Appendix, and are in some cases only briefly hinted in the main text.

\section{The RAndom Trip Model Definition}

The random trip mobility model is defined by the following framework.

\section{A. Trip, Phase, Path}

1) Domain: The domain $\mathcal{A}$ is a subset of $\mathbb{R}^{d}$, for some integer $d \geq 1$.

2) Phase: $I$ is a set of phases on $\mathbb{R}^{m}$, for some integer $m \geq 1$. A phase describes some state of the mobile, specific to the model. For example, it may indicate whether the mobile moves or pauses at a given time.

3) Path: $\mathcal{P}$ is a set of paths on $\mathcal{A}$. A path is a continuous mapping from $[0,1]$ to $\mathcal{A}$ that has a continuous derivative except maybe at a finite number of points (this is necessary to define the speed).

For $p \in \mathcal{P}, p(0)$ is the origin of $p, p(1)$ is its destination, and $p(u)$ is the point on $p$ attained when a fraction $u \in[0,1]$ of the path is traversed.

4) Trip: A trip is specified by a path $P_{n}$ and a duration $S_{n}$. The position $X(t)$ of the mobile at time $t$ is defined iteratively as follows. There is a set $T_{n} \in \mathbb{R}^{+}, n \in \mathbb{Z}_{+}$of transition instants, such that $T_{0} \leq 0<T_{1}<T_{2}<\ldots$. At time $T_{n}$, a phase $I_{n} \in I$, a path $P_{n} \in \mathcal{P}$ and a trip duration $S_{n} \in \mathbb{R}_{+}$are drawn according to some specified trip selection rule, specific to the model. The next transition instant is $T_{n+1}=T_{n}+S_{n}$ and the position of the mobile is

$$
X(t)=P_{n}\left(\frac{t-T_{n}}{S_{n}}\right) \quad \text { for } T_{n} \leq t \leq T_{n+1} .
$$

The trip selection rule is constrained to choose a path $P_{n}$ such that $P_{n}(0)=P_{n-1}(1)$. Further, we assume that, with probability 1 , the duration of the trip $S_{n}$ is positive (instantaneous transitions are not allowed).

Following a customary convention, whenever we consider a stationary realisation of node mobility, we extend the transition instants $T_{n}$ to the entire line $\mathbb{R}$, and enumerate them as $\ldots<$ $T_{-1}<T_{0} \leq 0<T_{1}<\ldots$. In these cases, 0 is an arbitrary time.

5) Default Initialisation Rule: At time $t=0$, a phase, path, position on the path, and remaining time until the next transition are drawn according to some specified initialisation rule. We define as a default initialisation rule that which takes time 0 as the first transition instant $\left(T_{0}=0\right)$, and selects a phase, path and trip duration according to the trip selection rule. The default initialisation rule has been used in simulations of many random mobility models (e.g. classical random waypoint).

We introduce additional assumptions. Some of the assumptions are either trivial to verify or always hold in real world, while some are crucial to guarantee stability of the random trip model and may not be always trivial to verify. This is discussed more concretely in Section II-D. In any case, the following assumptions accommodate a broad class of random mobility models.

\section{B. Conditions on Phase and Path}

(H1) $Y \equiv\left(Y_{n}\right)_{n=0}^{+\infty}$ with $Y_{n}:=\left(I_{n}, P_{n}\right)$ defined as a couple of phase and path is a Markov chain on $I \times \mathcal{P}$.

(H2) The chain $Y$ is Harris recurrent [19, Section III.9]: There exists a set $R \in I \times \mathcal{P}$, a probability measure $\varphi$ with support on $I \times \mathcal{P}$, a number $\beta \in(0,1)$, and an integer $n_{0} \geq 1$ such that the following two conditions hold

(i) $\quad \mathbb{P}_{y}\left(Y_{n} \in R\right.$ for some $\left.n \geq 1\right)=1$ for all $y \in I \times \mathcal{P}$

(ii) $\quad \mathbb{P}_{y}\left(Y_{n_{0}} \in B\right) \geq \beta \cdot \varphi(B)$ for all $y \in R$ and any measurable $B$ in $I \times \mathcal{P}$.

Here we use the notation $\mathbb{P}_{y}(\cdot)=\mathbb{P}\left(\cdot \mid Y_{0}=y\right), y \in I \times \mathcal{P}$.

Condition i implies that $R$ is a recurrent set of the chain. Condition ii says that $R$ is a "regeneration set" in the sense that the conditional probability that the chain hits a set $B$, after $n_{0}$ transitions from $y \in R$, is $\varphi(B)$ with probability $\beta$, where $\varphi(\cdot)$ is independent of $y$.

Condition $\mathrm{H} 2$ ensures the chain $Y$ has a unique stationary measure (up to a multiplicative constant) $\pi^{0}$ defined by

$$
\pi^{0}(A)=\int_{I \times \mathcal{P}} P(y, A) \pi^{0}(d y)
$$

where $P(y, A):=\mathbb{P}\left(Y_{1} \in A \mid Y_{0}=y\right)$ is the transition semigroup of the chain.

(H3) The chain $Y$ is positive Harris recurrent, i.e. $\mathrm{H} 2$ holds and the number of transitions between successive visits to the set $R$ has a finite expectation.

Condition H3 implies the invariant measure $\pi^{0}$ is such that $\pi^{0}(I \times P)<+\infty$, so that it can be normalised to a probability distribution.

\section{Conditions on Trip Duration}

(H4) Three hypotheses: 
(i) The distribution of a trip duration $S_{n}$, given the phase $I_{n}$ and path $P_{n}$, is independent of any other past and $n$. Formally, we have

$$
\begin{aligned}
& \mathbb{P}\left(S_{n} \leq s \mid Y_{n}=y, Y_{n-1}, S_{n-1}, \ldots\right) \\
= & \mathbb{P}\left(S_{n} \leq s \mid Y_{n}=y\right):=F(y, s), \text { for all } n \in \mathbb{Z} .
\end{aligned}
$$

We assume that for all $y \in I \times \mathcal{P}, F(y, s)$ is a non defective probability distribution, that is $\lim _{s \rightarrow+\infty} F(y, s)=1$, for all $y \in$ $I \times \mathcal{P}$. Note that in general the trip duration $S_{n}$ is dependent on the path $P_{n}$.

(ii) Each trip takes a strictly positive time, i.e.

$$
\mathbb{P}\left(S_{n}>0 \mid Y_{n}=y\right)=1 \text {, all } n \geq 1 \text { and } \pi^{0} \text { almost all } y .
$$

This condition is always true in reality.

(iii) The Markov renewal process $\left(Y_{n}, S_{n}\right)_{n=0}^{\infty}$ is non arithmetic, i.e. there exists no $d \geq 0$ and some "shift" function $g: I \times \mathcal{P} \rightarrow[0, d)$ such that given $Y_{0}=y, S_{0}$ takes values on the set $g(y)+d \mathbb{Z}_{+}$, for $\pi^{0}$ almost all $y$.

This assumption is automatically true if there is a subset $Y^{0} \in I \times \mathcal{P}$ of strictly positive $\pi^{0}$ probability such that, given $Y_{n} \in Y^{0}$, the distribution of $S_{n}$ has a density, i.e. $F(y, s)=$ $\int_{0}^{s} f(y, t) d t$, for some function $f(\cdot)$, and $y \in Y^{0}$.

Condition H4.iii is needed to state the convergence in distribution to a time-stationary distribution as specified in Theorem 6-item ii, for sample paths initialised at $t=0$ as specified by the default initialisation rule (see item 5 in Section II-A) ${ }^{1}$

\section{How to Verify the Conditions in Practise?}

Condition $\mathrm{H} 1$ is a structural assumption on the trip selection over time and is easy to verify; the same holds for H4.i and H4.ii.

Condition H4.iii is true as soon as the trip duration has a density, for a non negligible subset of paths and phases. In practise, trip durations either have a density or are mixtures of constants. It is sufficient that, for some (non negligible) subset of path and phase conditions, the trip duration has a density. For example, H4.iii is true for a model with pauses if either the pause duration, or the (non pause) trip duration has a density.

Conditions $\mathrm{H} 2$ and $\mathrm{H} 3$ are stronger. They essentially say that the Markov chain of system states, sampled at trip endpoints, is stable, in a strong sense. The technical difficulty here is that, for many examples, we have a Markov chain on a non countable state space, for which stability conditions are mathematically complicated. However, it helps to think that for random trip with a countable state space $I \times \mathcal{P}$, conditions $\mathrm{H} 2$ and $\mathrm{H} 3$ simply mean positive recurrence. For a finite state space, they even more simply mean that the state space is connected.

We next show that conditions H1-H4 are verified by many random mobility models.

\footnotetext{
${ }^{1}$ Condition H4.iii is not needed for existence and uniqueness of a time stationary distribution (Theorem 6-item i, Section IV) and one can indeed construct time-stationary sample paths of mobility when H4.iii does not hold by appropriate initialisation.
}

\section{Notation Used in Section II}

- $\mathcal{A} \subset \mathbb{R}^{d}:$ model domain, connected and bounded

- $d(m, n)$ length of shortest path in $\mathcal{A}$ from $m \in \mathcal{A}$ to $n \in \mathcal{A}$; if $\mathcal{A}$ is convex $d(m, n)=\|m-n\|$

- $T_{n}: n$th transition time, at which a new trip is defined

- $I_{n} \in I, M_{n} \in \mathcal{A}, P_{n} \in \mathcal{P}, S_{n} \in(0, \infty)$ : phase, starting point, path, trip duration for the trip indexed by $n$. The first trip has index $n=0$.

- $I(t) \in I, M(t) \in \mathcal{A}, P(t) \in \mathcal{P}, S(t) \in(0, \infty), X(t) \in \mathcal{A}$ : phase, starting point, path, trip duration for the trip used by mobile at time $t$, location at time $t$. $X\left(T_{n}\right)=M_{n}$ and if $T_{n} \leq t<T_{n+1}$ then $I(t)=I_{n}, M(t)=M_{n}$ and $S(t)=S_{n}$. - $U(t) \in[0,1]$ : fraction of the current trip that was already traversed. Thus $U(t) S(t)$ is the time elapsed on the current trip and the location of the mobile at time $t$ is $X(t)=p(U(t))$, with $p=P(t)$. We assume that the trip is done at a speed proportional to the default speed of the path, i.e. if $T_{n} \leq t<T_{n+1}$ then $U(t)=\frac{t-T_{n}}{T_{n+1}-T_{n}}=\frac{t-T_{n}}{S_{n}}$

- It follows that the speed vector of the mobile at a time $t$ that is not an end of trip is $\vec{V}(t)=\frac{1}{S(t)} \frac{\partial}{\partial u} p(U(t))$, with $p=P(t)$ and the numerical speed is $V(t)=\|\vec{V}(t)\|$.

- For some random variable $Z, \mathbb{E}^{0}(Z)$ is the "Palm expectation", which can be interpreted as the expectation, conditional to the event that a transition occurs at time 0 , when the system has a stationary regime. $\mathbb{E}^{0}$ denotes the event average viewpoint [4], [17]. For example $\mathbb{E}^{0}\left(S_{0}\right)=\mathbb{E}^{0}(S(0))$ is the average trip duration; in contrast, when the system has reached steadystate, $\mathbb{E}(S(0))=\mathbb{E}(S(t))$ is the average duration of a trip, seen from an observer who samples the system at an arbitrary point in time. Both are usually different because the observer is more likely to sample a large trip duration.

- In order to simplify notation and at no expense of ambiguity, for a rightcontinuous process $X(t), t \in \mathbb{R}$, and appropriately defined function $f(\cdot)$, we write $\mathbb{E}\left(f\left(X_{0}\right)\right)$ for the Palm expectation $\mathbb{E}^{0}(f(X(0)))$; here $X_{n}:=X\left(T_{n}\right)$ with $T_{n}$ a trip transition instant.

- We say a property holds for $\pi^{0}$ almost all $y$, if it holds for all $y \in I \times \mathcal{P}$, but maybe not for some $y$ that lies in a set of zero $\pi^{0}$ measure.

\section{EXAMPLES}

We give a non exhaustive catalogue of example random mobility models and show they are all random trip models.

\section{A. Classical Random Waypoint With Pauses}

This is the classical random waypoint model. $\mathcal{A}$ is assumed to be convex ( $\mathcal{A}$ is a rectangle or a disk in [12], [10]). Paths are straight line segments: $p(u)=(1-u) m_{0}+u m_{1}$ for the segment with endpoints $m_{0}$ and $m_{1}$. Pauses are special cases of paths, when endpoints are equal: $p(u)=m_{0}$. There are two phases $I=\{$ pause, move $\}$. At a transition instant, the trip selection rule alternates the phase from pause to move or vice versa. If the new phase is pause, the trip duration $S_{n}$ is drawn from the distribution $F_{\text {pause }}^{0}(s)$; the path $P_{n}$ is a pause at the current point. If the new phase is move, the trip selection rules picks a point $M_{n+1}$ at random uniformly in $\mathcal{A}$, and a numerical speed $V_{n}$ according to the density $f_{V}^{0}(v)$. A classical choice (uniform speed) is $f_{V}^{0}(v)=\frac{1}{v_{\max }-v_{\min }} 1_{\left\{v_{\min }<v<v_{\max }\right\}}$. The trip duration is then $S_{n}=\frac{\left\|M_{n+1}-M_{n}\right\|}{V_{n}}$ and the path $P_{n}$ is the segment $\left[M_{n}, M_{n+1}\right]$. The default initialisation rule starts the model at the beginning of a pause, at a location uniformly chosen in $\mathcal{A}$.

Theorem 1: The random waypoint with pauses is a random trip model.

Proof. H1 and H4 obviously hold. By Theorem 2 shown next it is sufficient to consider the model without pauses. The driving chain is now $Y_{n}=\left(P_{n}\right)=\left(M_{n}, M_{n+1}\right)$ and is indeed Markov. Take as recurrent set $R:=\mathcal{A}^{2}$ so that condition $\mathrm{H} 2$.i obviously holds. The paths are selected such that $P_{n}$ and $P_{m}$ are independent for $|n-m| \geq 2$. It follows that for any $n_{0} \geq 2$ and any $y \in R$, 


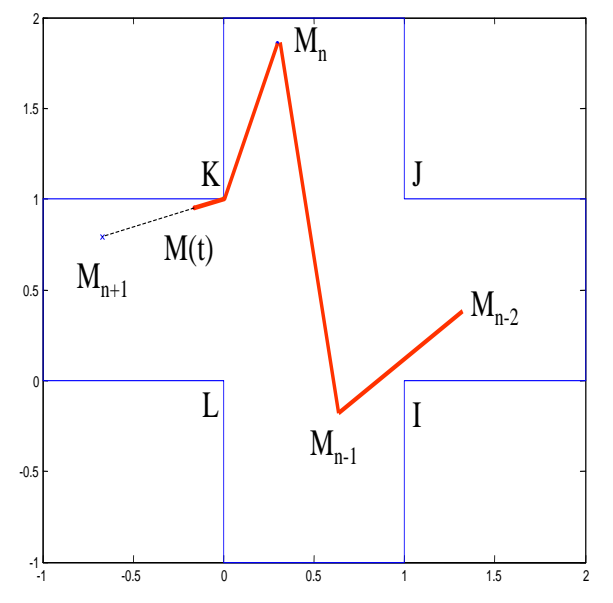

Fig. 3. Random Waypoint on a non convex domain (Swiss Flag). A trip is the shortest path inside the domain from a waypoint $M_{n}$ to the next. Waypoints $M_{n}$ are drawn uniformly in the domain. In figure, the shortest path $M_{n}, M_{n+1}$ has two segments, with a breakpoint at $K$; the shortest paths $M_{n-1}, M_{n}$ and $M_{n-2}, M_{n-1}$ have one segment each. $M(t)$ is the current position.

$$
\mathbb{P}_{y}\left(Y_{n_{0}} \in A_{1} \times A_{2}\right)=\operatorname{Unif}\left(A_{1}\right) \operatorname{Unif}\left(A_{2}\right)
$$

where Unif is the uniform distribution on $\mathcal{A}$, defined by $\operatorname{Unif}(A)=\int_{A} d x / \int_{\mathcal{A}} d x$. This shows that H2.ii holds with $\varphi=$ Unif $\otimes$ Unif (product measure) and any $\beta \in(0,1)$. The recurrent set $R$ is visited at each transition, so H3 is indeed true.

This model is well known; its stationary properties are studied in [24], [12], [16]. However, even for this simple model our framework provides two new results: the proof of existence of a stationary regime, and a sampling algorithm for the stationary distribution over general areas that does not require the computation of geometric integrals.

\section{B. Adding Pauses to a Model}

Assume we have a random trip model $\mathcal{M}$ with phases $I_{n}$ and paths $P_{n}$. We can add pauses to this model and obtain a new model $\mathcal{M}^{\prime}$ as follows. At the end of the $n$th trip, a pause time $S_{n}^{\prime}$ is drawn at random, depending only (possibly) on the current trip and phase. This means that the pause duration at the end of the $n$th trip, conditional on all past, depends only on $P_{n}$ and $I_{n}$.

In $\mathcal{M}^{\prime}$ we have phases $I_{n}^{\prime}$ and paths $P_{n}^{\prime}$ given by (for all $k \in \mathbb{N})$ :

$$
\left\{\begin{array}{l}
I_{2 k}^{\prime}=\left(\text { move }, I_{k}\right), I_{2 k+1}^{\prime}=\left(\text { pause }, I_{k}\right) \\
P_{2 k}^{\prime}=P_{k}, P_{2 k+1}^{\prime}=\operatorname{constant}\left(M_{k+1}\right)
\end{array}\right.
$$

where constant $(m)$ is the path that remains entirely at point $M$ (i.e. $\operatorname{constant}(m)(u)=m$ for $0 \leq u \leq 1)$.

Theorem 2: If $\mathcal{M}$ is a random trip model, then $\mathcal{M}^{\prime}$ is also a random trip model.

Proof. It is straightforward to show that assumptions $\mathrm{H} 1$ and $\mathrm{H} 4$ hold. We now show $\mathrm{H} 2$.i. Let $R$ be a regeneration set for model $\mathcal{M}$, which by hypothesis exists. Let

$$
R^{\prime}=\{\text { move }, \text { pause }\} \times R
$$

Let $Z_{n} \in\{$ move,pause $\}$ be the sequence that alternates between move and pause, and indicates whether the $n$th trip is a pause or not. The driving chain in model $\mathcal{M}^{\prime}$ is $Y_{n}^{\prime}$. If $Z_{0}=$ move (this is implicitly assumed in Equation (2)) then $Y_{2 k}^{\prime}=\left(\right.$ move $\left., I_{k}, P_{k}\right)$ and thus

$$
\begin{aligned}
& \mathbb{P}_{(\text {move }, i, p)}\left(Y_{n}^{\prime} \in R^{\prime} \text { for some } n\right) \\
& \quad \geq \mathbb{P}_{(\text {move }, i, p)}\left(Y_{2 k}^{\prime} \in R^{\prime} \text { for some } k\right) \\
& \quad=\mathbb{P}_{(i, p)}\left(Y_{k} \in R \text { for some } k\right)=1
\end{aligned}
$$

and similarly

$$
\begin{aligned}
& \mathbb{P}_{(\text {pause }, i, p)}\left(Y_{n}^{\prime} \in R^{\prime} \text { for some } n\right) \\
& \quad \geq \mathbb{P}_{(\text {pause }, i, p)}\left(Y_{2 k+1}^{\prime} \in R^{\prime} \text { for some } k\right) \\
& \quad=\mathbb{P}_{(i, p)}\left(Y_{k} \in R \text { for some } k\right)=1
\end{aligned}
$$

which shows H2.i.

To show H2.ii, let $n_{0}, \beta, \varphi$ be such that $\mathbb{P}_{(i, p)}\left(Y_{n_{0}} \in B\right) \geq$ $\beta \cdot \varphi(B)$ for all $y \in R$ and any measurable $B$ in $I \times \mathcal{P}$. Define the probability measure on $\{$ move, pause $\} \times I \times \mathcal{P}$ by $\varphi^{\prime}(\{$ move $\} \times B)=\varphi(B)$ and $\varphi^{\prime}(\{$ pause $\} \times B)=0$. We have, for $B^{\prime}=\{$ move $\} \times B$ :

$\mathbb{P}_{(\text {move }, i, p)}\left(Y_{2 n_{0}}^{\prime} \in B^{\prime}\right)=\mathbb{P}_{(i, p)}\left(Y_{n_{0}} \in B\right) \geq \beta \varphi(B)=\beta \varphi^{\prime}\left(B^{\prime}\right)$

and for $B^{\prime}=\{$ pause $\} \times B$ :

$$
\mathbb{P}_{(\text {move }, i, p)}\left(Y_{2 n_{0}}^{\prime} \in B^{\prime}\right)=0=\beta \varphi^{\prime}\left(B^{\prime}\right)
$$

which shows H2.ii.

\section{Random Waypoint on General Connected Domain}

This is a variant of the classical random waypoint (Example III-A), where we relax the assumption that $\mathcal{A}$ is convex, but assume that $\mathcal{A}$ is a connected domain over which a uniform distribution is well defined. For two points $m, n$ in $\mathcal{A}$, we call $d(m, n)$ the distance from $m$ to $n$ in $\mathcal{A}$, i.e. the minimum length of a path entirely inside $\mathcal{A}$ that connects $m$ and $n . \mathcal{P}$ is the set of shortest paths between endpoints. The trip selection rule picks a new endpoint uniformly in $\mathcal{A}$, and the next path is the shortest path to this endpoint. If there are several shortest paths, one of them is randomly chosen according to some probability distribution on the set of shortest paths. The set of phases is $I=\{$ pause, move $\}$. This model fits in our framework for the same reasons as the former example.

1) Swiss Flag: The model is random waypoint on particular non-convex domain defined by the cross section as in Figure 3.

2) City Section: This is a special case of random waypoint on a non convex domain. The domain is the union of the segments defined by the edges of the space graph (e.g. Figure 1). Arbitrary numeric speeds can be assigned to edges of the graph. The "distance" from one location to another is the travel time.

\section{Restricted Random Waypoint}

This model was originally introduced by Blažević et al [6] in a special form described in Figure 4, in order to model intercity examples. We define it more generally as follows. 


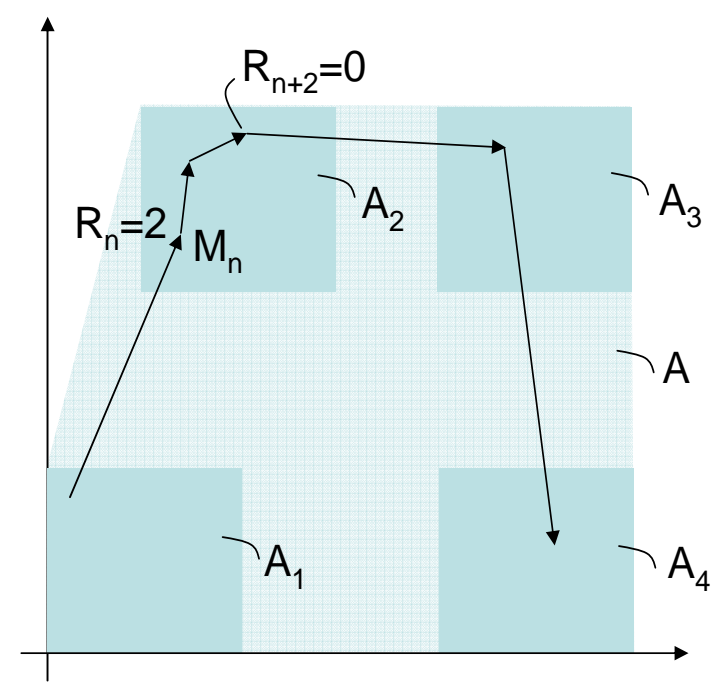

Fig. 4. A restricted random waypoint on a plane with four squares as subdomains. This model was introduced in [6] to simulate a wide-area routing protocol. It was used as an idealised view of four towns represented by squares. A mobile moves according to random waypoint within a square for a random number of visits and then picks a point uniformly at random in another randomly chosen square as a destination. The figure shows a sample path of the mobile movement. The speed on the trip is chosen according to a distribution that depends on the origin and destination squares.

The trip endpoints are selected on a finite set of subdomains $\mathcal{A}_{i} \in \mathbb{R}^{d}, i \in \mathcal{L}$. The domain $\mathcal{A}$ is a convex closure of the subdomains $\mathcal{A}_{i}, i \in \mathcal{L}$. The trip selection rule is described as follows. To simplify, we first consider a node movement with no pauses. Suppose the node starts from a point $M_{n}$ chosen uniformly at random on a subdomain $i$. The node picks the number of trips $r$ to undergo with trip endpoints in the subdomain $i$ from a distribution $F_{i}(\cdot)$. The next subdomain is drawn from the distribution $Q(i, \cdot)$. At each trip transition, the node decrements $r$ by 1 , as long as $r>0$, else it sets $r$ to a random sample from the distribution $F_{j}(\cdot)$. Then, if $r=0$, the current subdomain is set to $j$ and the next subdomain to a sample from $Q(j, \cdot)$. The trip destination is chosen uniformly at random on $\mathcal{A}_{i}$ if $r>0$, else uniformly at random on $\mathcal{A}_{j}$. This process repeats. The model is extended to accommodate pauses in a straightforward manner by inserting pauses at the trip transition instants.

The phase is $I_{n}=(i, j, r, \phi)$, where $i$ and $j$ are respectively, the current and next subdomain, $r$ is the number of trips with both endpoints in the subdomain $i$, and $\phi \in\{$ move,pause $\}$. Given a phase $I_{n}=(i, j, r$, move $)$, the path $P_{n}$ is the line segment $\left[M_{n}, M_{n+1}\right]$, with $M_{n}$ uniformly distributed on $\mathcal{A}_{i}$ and $M_{n+1}$ uniformly distributed on $\mathcal{A}_{i}$ or $\mathcal{A}_{j}$, for $r>0$ and $r=0$, respectively.

Theorem 3: Assume that (i) $Q$ is an irreducible transition matrix, and (ii) the number of trips within a subdomain has a finite expectation, i.e. $\sum_{n=0}^{\infty}\left(1-F_{i}(n)\right)<+\infty$, for all $i \in \mathcal{L}$. Then, the restricted random waypoint is a random trip model.

Proof (given in Appendix) derives from known ergodicity results for Markov chains on countable state spaces.

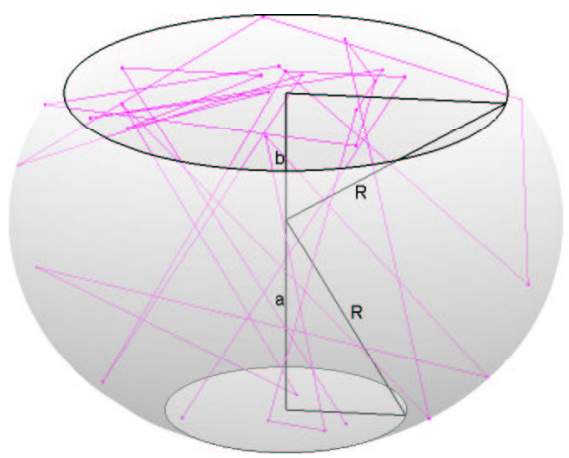

Fig. 5. Fish in a bowl is a restricted random waypoint. $\mathcal{A}$ is the volume of the sphere comprised between two horizontal planes. Waypoints are on the boundary $\mathcal{A}_{1}$ of $\mathcal{A}$.

In addition to the model in Figure 4, we give two particular examples of the restricted random waypoint model.

1) Fish in a Bowl: The model is restricted random waypoint on the domain defined by the volume of the bowl, as in Figure 5. The waypoints are restricted to the subset $\mathcal{A}_{1}$ of the domain $\mathcal{A}$, where $\mathcal{A}_{1}$ is the set of the points on the bowl's surface (see Figure 5). The set of phases is $I=\{$ pause, move $\}$.

2) Space Graph: We defined this model in Section I. It is a special case of restricted random waypoint with $\mathcal{A}=$ the space graph and $\mathcal{A}_{1}=$ the set of vertices. Note that it differs from the City Section in that the waypoints are restricted to be vertices. The set of phases is $I=\{$ pause,move $\}$.

Note that all models III-A to III-D. 2 and III-E are special cases of the restricted random waypoint, with $L=1, r=0$, and $\mathcal{A}_{1}=\mathcal{A}$ for examples III-A to III-C.2, $\mathcal{A}$ a strict subset of $\mathcal{A}$ for examples III-D.1 and III-D.2. Note that the subdomains $\mathcal{A}_{\ell}$ may be convex as in Figure 4 or not as in Figure 5.

\section{E. Random Waypoint on Sphere}

Here $\mathcal{A}$ is the unit sphere of $\mathbb{R}^{3} . \mathcal{P}$ is the set of shortest paths plus pauses. The shortest path between two points is the shortest of the arcs on the great circle that contains the two points. If the two points are on the same great circle diameter, the two arcs have same length (this occurs with probability 0 ). The trip transition rule picks a path endpoint uniformly on the sphere, and the path is the shortest path to it (if there are two, one is chosen with probability 0.5 ). The set of phases is $I=$ $\{$ pause, move $\}$. The numerical speed is chosen independently. Initially, a point is chosen uniformly.

This model is in fact a special case of the random waypoint on a connected, non convex domain. However, we mention it separately as it enjoys special properties (the stationary location is uniform, unlike for the random waypoint models described earlier).

\section{F. Random Walk on Torus}

This model is called a random waypoint on a torus in [18]. It is used primarily because of its simplicity: unlike for the random waypoint, the distribution of location and speed at a random instant are the same as at a transition instant, as we show later. 


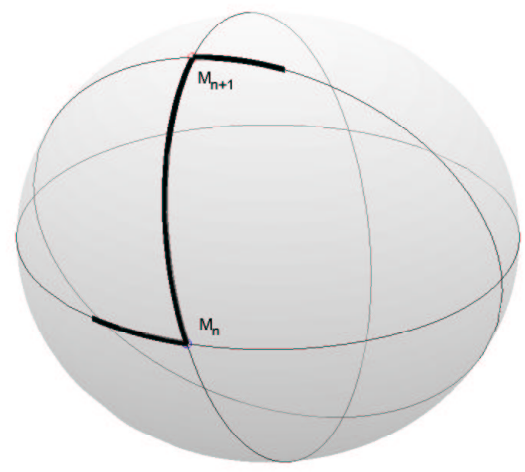

Fig. 6. Random waypoint on a sphere.

The domain $\mathcal{A}$ is the rectangle $\left[0, a_{1}\right] \times\left[0, a_{2}\right]$. Paths are wrapped segments, defined as follows. The trip selection rule chooses a speed vector $\vec{V}_{n}$ and a trip duration $S_{n}$ independently, according to some fixed distributions. Choosing a speed vector $\vec{V}_{n}$ is the same as choosing a direction of movement and a numerical speed. The mobile moves from the endpoint $M_{n}$ in the direction and at the numeric speed given by the speed vector. When it hits the boundary of $\mathcal{A}$, say for example at a location $\left(x_{0}, a_{2}\right)$, it is wrapped to the other side, to location $\left(x_{0}, 0\right)$, from where it continues the trip (Figure 7). Let $w$ : $\mathbb{R}^{2} \rightarrow \mathcal{A}$ be the wrapping function:

$$
\left(\begin{array}{c}
x \\
y
\end{array}\right) \mapsto w\left(\begin{array}{c}
x \\
y
\end{array}\right)=\left(\begin{array}{cc}
x & \bmod a_{1} \\
y & \bmod a_{2}
\end{array}\right) .
$$

The path $P_{n}$ (if not a pause) is defined by $\left(M_{n}, \vec{V}_{n}, S_{n}\right)$, such that $P_{n}(u)=w\left(M_{n}+u S_{n} \vec{V}_{n}\right)$. Note that wrapping does not modify the speed vector (Figure 7). After a trip, a pause time is drawn independent of all past from some fixed distribution. Initially, the first endpoint is chosen in $\mathcal{A}$ according to some arbitrary distribution. As we see later, the distribution of endpoint tends to uniform distribution (when sampled at transition instants).

For $a_{1}=a_{2}=1$, and if there are no pauses, the sequence $M_{0}, \ldots, M_{n}, \ldots$ is a random walk on the torus, in the sense that $M_{n}=M_{0} \oplus \vec{U}_{0} \oplus \vec{U}_{1} \oplus \cdots \oplus \vec{U}_{n-1}$ where $\oplus$ is addition modulo 1 (componentwise) and $\vec{U}_{n} \equiv S_{n} \vec{V}_{n}$. This is why this mobility model is itself called random walk.

Assumptions $\mathrm{H} 1$ and $\mathrm{H} 4$ are obviously satisfied by the random walk, with set of phases $I=\{$ pause, move $\}$. The other assumptions of the random trip model are satisfied modulo some mild assumption on distributions:

Theorem 4: Assume that the distribution of the speed vector $\vec{V}_{n}$ chosen by the trip selection rule has a density (with respect to the Lebesgue measure in $\mathbb{R}^{2}$ ). Further assume that either the distribution of trip durations or distribution of pause times have a density. The random walk on torus satisfies the random trip assumptions.

Remark. Note that we do not assume any form of symmetry for the direction of the speed vector, contrary to [21].

The proof is based on a sequence of lemmas that are displayed in the rest of this subsection. The first lemma characterises the node location at trip end points.
Lemma 1: In the random walk without pause, the sequence $M_{0}, \ldots, M_{n}, \ldots$ is a Harris recurrent Markov chain, with stationary distribution uniform on $\mathcal{A}$.

The asserted convergence is proved by using Erdös-TuránKoksma inequality [20, Theorem 1.21], which yields the following result:

Lemma 2: For any $m \in \mathcal{A}$,

$$
\lim _{n \rightarrow+\infty} \sup _{B}\left|\mu_{m}^{* n}(B)-\operatorname{Unif}(B)\right|=0
$$

where the supremum is over all product intervals in $\mathcal{A}$ and $\mu_{m}^{* n}$ is the conditional distribution of $M_{n}$ given $M_{0}=m$.

In order to apply the Erdös-Turán-Koksma inequality, we need the following auxiliary result.

Lemma 3: Let $X$ be a real random variable that is nonlattice. For $h \in \mathbb{Z}, h \neq 0$ :

$$
\left|\mathbb{E}\left(e^{2 i \pi h X}\right)\right|<1
$$

Proof. We apply the Cauchy Schwartz [13, Section 6.5p.132] inequality to the complex valued random variables $e^{2 i \pi h X}$ and 1 . We have

$$
\left|\mathbb{E}\left(e^{2 i \pi h X}\right)\right|^{2} \leq \mathbb{E}\left(\left|e^{2 i \pi h X}\right|^{2}\right)=1
$$

and equality implies that $e^{2 i \pi h X}=c$ a.s. for some constant $c \in \mathbb{C}$, and $X$ has to be lattice.

\section{G. Billiards}

This is similar to example III-F, but with billiards-like reflections instead of wrapping (Figure 7). The definition is identical to example III-F, with the wrapping function replaced by the billiards reflection function $b: \mathbb{R}^{2} \rightarrow \mathcal{A}$, defined by

$$
\left(\begin{array}{c}
x \\
y
\end{array}\right) \mapsto b\left(\begin{array}{c}
x \\
y
\end{array}\right)=\left(\begin{array}{l}
a_{1} b_{1}\left(\frac{x}{a_{1}}\right) \\
a_{2} b_{1}\left(\frac{y}{a_{2}}\right)
\end{array}\right)
$$

where $b_{1}: \mathbb{R} \rightarrow[0,1]$ is the 2-periodic function defined by

$$
b_{1}(x)=|x|, \text { for }-1 \leq x \leq 1 .
$$

Unlike the wrapping function, the billiards reflection may alter the speed vector (Figure 7). Therefore we make a difference between the unreflected speed vector $\vec{W}_{n}$ and the instant speed vector $\vec{V}(t)$ at time $t$. In the model without pause, the sequence of node locations $M_{0}, M_{1}, \ldots$ is a Markov chain, defined by

$$
M_{n+1}=b\left(M_{n}+\vec{U}_{n}\right), \quad n \geq 0,
$$

where $\vec{U}_{n}:=S_{n} \vec{W}_{n}$ is the driving sequence of i.i.d. random variables. The path $P_{n}$ (if not a pause) is defined by $\left(M_{n}, \vec{W}_{n}, S_{n}\right)$, such that $P_{n}(u)=b\left(M_{n}+u S_{n} \vec{W}_{n}\right)$.

The billiards is similar to the random walk on torus, but is not quite as simple ( $M_{n}$ is not a random walk). We need to impose that the speed vector has equal probability of going up or down [resp. left or right].

Definition 1: We say that a random vector $(X, Y)$ has a completely symmetric distribution iff $(-X, Y)$ and $(X,-Y)$ have the same distribution as $(X, Y)$. 

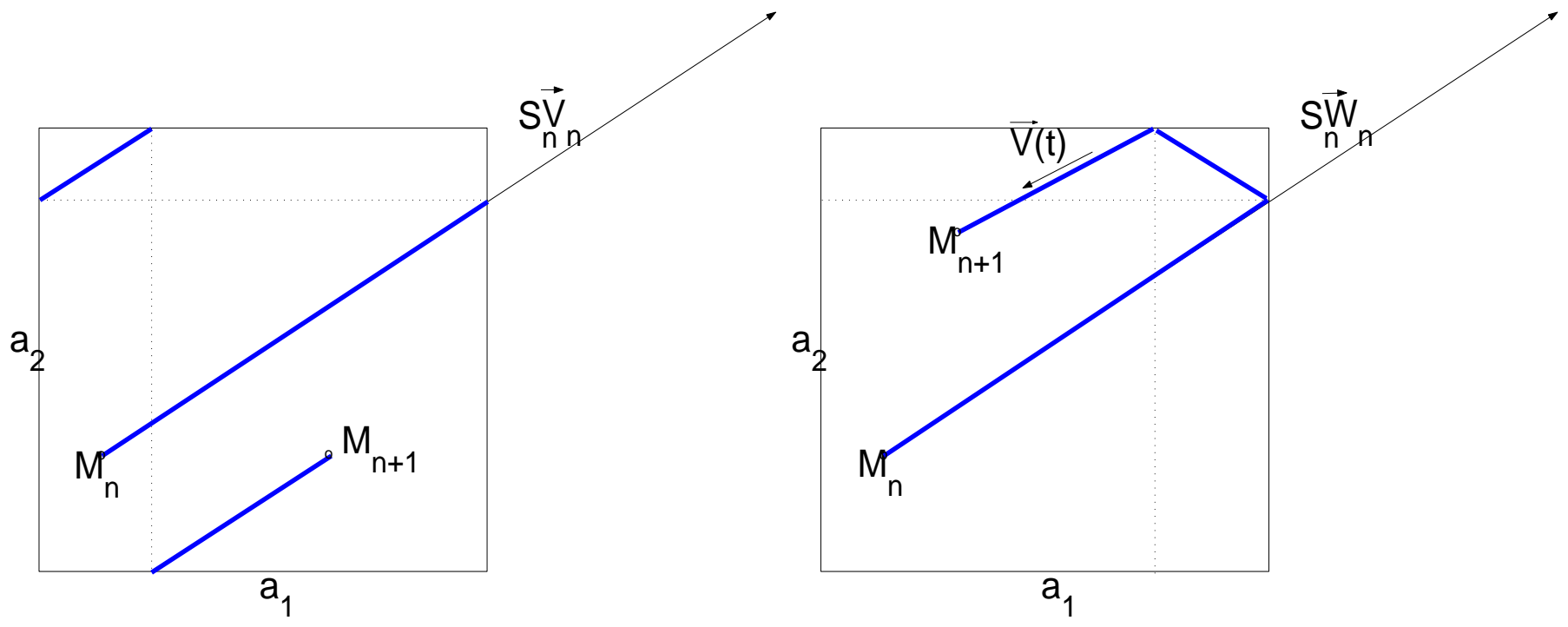

Fig. 7. Definition of random walk on torus (left) and billiards (right).

This is true for example if the direction of $\vec{W}$ is uniformly chosen on the unit circle, or if the two coordinates of $\vec{W}$ are independent and have even distributions. With this assumption, we have a similar result as for the random walk:

Theorem 5: Assume that the distribution of the speed vector $\vec{W}_{n}$ chosen by the trip selection rule has a density (with respect to the Lebesgue measure in $\mathbb{R}^{2}$ ) and is completely symmetric. Further assume that either the distribution of the trip durations or distribution of pause times have a density. The billiards satisfies the random trip assumptions.

Remark. Note that we need the complete symmetry of the speed vector for Lemma 4 to hold. Consider as counterexample a speed vector with density supported by the set $\left[0,0.1 a_{1}\right] \times \mathbb{R}$, i.e. it always goes to the right, by a little amount. After a few iterations, the sequence $M_{n}$ is always in the set $\left[0.9 a_{1}, a_{1}\right] \times\left[0, a_{2}\right]$, i.e. in a band on the right of the domain. So it cannot converge to a uniform distribution.

Proof. The proof is similar to that of Theorem 4, with Lemma 1 replaced by Lemma 4.

The theorem derives from a main lemma asserted here:

Lemma 4: In the billiards without pause (with the assumptions of Theorem 5), the sequence $M_{0}, \ldots, M_{n}, \ldots$ is a Harris recurrent Markov chain, with stationary distribution uniform on $\mathcal{A}$.

The proof in Appendixis by a pathwise reduction to a random walk on torus. Two auxiliary results are used in showing Lemma 4, which we present in the the rest of this section. First define, for any $m \in \mathbb{R}^{2}$ (possibly outside the area $\mathcal{A}$ ) the linear mapping $J_{m}$ that maps the non-reflected speed vector $\vec{w}$ to the speed vector $\vec{v}$ at the reflected location $b(m)$ $\left(J_{m}\right.$ is the differential of $b(\cdot)$ at point $m$ ) - see Appendix for details.

Lemma 5: For any non random point $m \in \mathcal{A}$ and vector $\vec{v} \in \mathbb{R}^{2}: b(m+\vec{v})=b\left(b(m)+J_{m}(\vec{v})\right)$.

Proof. It is enough to show the lemma in dimension 1. In this case, the result to prove is

$$
b_{1}(x+v)=b_{1}\left(b_{1}(x)+(-1)^{\lfloor x\rfloor} v\right)
$$

for any $x, v \in \mathbb{R}$. Both sides of the equation are 2-periodic in $x$, thus we can restrict to the cases $-1 \leq x<0$ and $0 \leq x<1$. In the former case, the equation is trivial. In the latter, it becomes $b_{1}(x+v)=b_{1}(-x-v)$, which is true because $b_{1}(\cdot)$ is even.

Lemma 6: For any $m \in \mathbb{R}^{2}$ :

$$
b(m)=b\left(w_{2}(m)\right)
$$

Proof. Each coordinate of $b(\cdot)$ is 2-periodic and the the wrapping $w_{2}(\cdot)$ is a translation by integer multiples of 2 .

\section{Time Stationarity AND CONVERGEnCE}

The state of a mobile node at time $t$ is described by the continuous-time Markov process

$$
\Phi(t):=\left(Y(t), S(t), S^{-}(t)\right),
$$

which takes values on the state space $I \times \mathcal{P} \times \mathbb{R}_{+}^{2}$. Here $S(t)$ is the duration of the trip at time $t$ and $S^{-}(t)$ is the elapsed time on the trip at time $t$. The random trip model definitions introduced in Section II imply $\Phi$ is a Markov renewal process. The following is the main stability result:

Theorem 6: For the random trip model specified by H1-H4 in Section II:

(i) There exists a time-stationary distribution $\pi$ for $\Phi$ if and only if $\mathbb{E}^{0}\left(S_{0}\right)$ is finite. Whenever $\pi$ exists, it is unique and given by:

$$
\pi(B)=\frac{\mathbb{E}^{0}\left(\int_{0}^{T_{1}} 1_{\Phi(s) \in B} d s\right)}{\mathbb{E}^{0}\left(S_{0}\right)}, B \in I \times \mathcal{P} \times \mathbb{R}_{+}^{2} .
$$

(ii-a) If $\mathbb{E}^{0}\left(S_{0}\right)$ is finite, then from $\pi^{0}$ almost any trip initiated at time $0, \Phi(t)$ converges in distribution to $\pi$, as $t \rightarrow+\infty$.

(ii-b) Else, if $\mathbb{E}^{0}\left(S_{0}\right)=+\infty$, then

$$
\lim _{t \rightarrow+\infty} \mathbb{P}_{y}(\Phi(t) \in A) \rightarrow 0,
$$


for any set $A$ in $I \times \mathcal{P} \times \mathbb{R}_{+}^{2}$ such that

$$
\mathbb{E}^{0}\left(\int_{0}^{T_{1}} 1_{\Phi(s) \in A} d s\right)<+\infty .
$$

Comment 1. The convergence result ii follows from the Markov renewal theorem [1]. We note that the result holds under assumption that the driving chain $Y$ is only Harris recurrent, not necessarily positive Harris recurrent. If the driving chain $Y$ is null-recurrent, i.e. the mean number of transitions between successive visits to regeneration sets is infinite, then it still may be that $\mathbb{E}^{0}\left(S_{0}\right)$ is finite and that the asserted limit hold. Similar convergence results are known for a positive Harris recurrent Markov process in continuous time, under a condition on the distribution of the regeneration epochs. See for instance [2, Proposition 3.8] for a convergence in total variation.

Comment 2. The item ii-b formalises the reported "harmfulness" of the random waypoint. It says that for a random trip model, if the mean trip duration $\mathbb{E}^{0}\left(S_{0}\right)$ is infinite, then the process $\Phi$ is in fact null-recurrent. The asserted convergence to 0 was originally found for node numeric speed [25].

Comment 3. The conditions introduced in [7] are sufficient conditions for $\mathrm{H} 1-\mathrm{H} 3$ to hold. Condition H4.iii is new and is needed for the asserted convergence in item ii, not for item i.

Corollary 1: For examples III-A to III-E, there is a stationary regime if and only if the pause time and inverse speed (sampled at a transition) have a finite expectation. For examples III-F and III-G the condition is that the pause time and trip duration (sampled at a transition) have a finite expectation.

\section{TIME-STATIONARy Distributions}

For a perfect simulation, all we need is to sample from the time stationary distribution of the process state. The state of the process is the phase $I(t)$, the path $P(t)$, the trip duration $S(t)$ and where on trip $U(t)$. In this section we give a simple representation of the time stationary distribution of this process state for any random trip model. In the next sections we will apply it to the various examples introduced earlier.

Our representation relates this distribution to the stationary distribution $\pi^{0}$ of the Markov chain $Y_{n}=\left(I_{n}, P_{n}\right)$ of phase and path sampled at transition instants, and to the mean trip duration $\bar{\tau}(y):=\int_{0}^{+\infty} s F(y, d s)$ given that the phase and path is $y$.

Theorem 7: Assume the condition for existence and uniqueness of a stationary distribution in Section IV of Part I is satisfied. The time stationary distribution of the process state at an arbitrary time $t$ is given by the following.

1) Phase and Path: Let $Y(t)=(I(t), P(t))$.

$$
d \mathbb{P}(Y(t)=y)=\frac{\bar{\tau}(y)}{\int_{\mathcal{Y}} \bar{\tau}(x) \pi^{0}(d x)} \pi^{0}(d y) .
$$

2) Trip duration, given phase and path:

$$
d \mathbb{P}(S(t)=s \mid Y(t)=y)=\frac{s}{\bar{\tau}(y)} F(y, d s) .
$$

3) Fraction of time elapsed on the trip: $U(t)$ is independent of $(I(t), P(t), S(t))$ and is uniform on $[0,1]$.
Notation Used in Section VI

- $Q(i, j)$ : probability that next subdomain is $\mathcal{A}_{j}$ given current subdomain is $\mathcal{A}_{i} \cdot q^{0}$ is the unique stationary probability of $Q$ given by $q^{0} Q=q^{0}$.

- For $r \in \mathbb{N}_{+}, F_{i}(r)$ is the probability that the number of consecutive trips within subdomain $\mathcal{A}_{i}$ is smaller or equal $r$, with $r \geq 0 . \bar{R}_{i}=\sum_{r>0} \bar{F}_{i}(r)$, with $\bar{F}_{i}(r)=1-F_{i}(r)$, is the expected number of consecutive trips within subdomain $\mathcal{A}_{i}$.

- $\bar{\Delta}_{i, j}$ is the average distance in $\mathcal{A}$ for two points chosen uniformly in $\mathcal{A}_{i}$ and $\mathcal{A}_{j} . \Delta_{i, j}$ is an upper bound on the distance in $\mathcal{A}$ between two points in $\mathcal{A}_{i}$ and $\mathcal{A}_{j}$.

- $f_{V \mid \ell}^{0}(v)$ is the Palm (= at a transition instant) distribution of speed, given that phase is $\ell=(i, j, r$, move $) ; \omega_{i, j}=\mathbb{E}^{0}\left(\frac{1}{V_{0}} \mid I_{n}=(i, j, r\right.$, move $\left.)\right)$ is the event average of the inverse of the speed chosen for a trip from subdomain $\mathcal{A}_{i}$ to $\mathcal{A}_{j}$. We have $\omega_{i, j}=\int_{0}^{\infty} \frac{1}{v} f_{V \mid i, j, r, m o v e}(v) d v$, assumed to be independent of $r$. - $F_{S \mid \ell}^{0}(s)$ is the Palm (= at a transition instant) distribution of pause time, given that phase is $\ell=(i, j, r$, pause $) ; \tau_{i, j}=\mathbb{E}^{0}\left(S_{0} \mid I_{0}=(i, j, r\right.$, pause $\left.)\right)$ is the expected pause time of a pause, given that origin and destination subdomains are $\mathcal{A}_{i}$ to $\mathcal{A}_{j}$. We have $\tau_{i, j}=\int_{0}^{\infty} s F_{S \mid i, j, \text { r.pause }}^{0}(d s)$, assumed to be independent of $r$.

Note that the factor $\int_{y} \bar{\tau}(y) \pi^{0}(d y)$ in the denominator of item 1 is the mean trip duration, and the stability condition in Part I, Section IV is precisely that it is finite.

Special Case: Independent Pauses. In many examples with pauses, the set of phases is reduced to $\{$ pause,move $\}$, the model alternates between these two, and $\pi^{0}\left(I_{0}=i\right)=0.5$ for $i=$ pause or move. Define $\bar{\tau}_{\text {pause }}$ [resp. $\bar{\tau}_{\text {move }}$ ] as the mean pause duration (sampled at trip endpoints) [resp. mean trip duration for a trip that is not a pause]. It follows from item 1 that

$$
\mathbb{P}(I(t)=\text { pause })=\frac{\bar{\tau}_{\text {pause }}}{\bar{\tau}_{\text {pause }}+\bar{\tau}_{\text {move }}}
$$

and $\mathbb{P}(I(t)=$ move $)=1-\mathbb{P}(I(t)=$ pause $)$.

\section{Vi. Application to Examples A to D}

In all of this section, we assume that the condition for stationarity in Part I is satisfied. We focus on restricted random waypoint on general connected area, since examples A to D are special cases of it.

\section{A. Time Stationary Distributions}

A direct application of Theorem 7 gives the time stationary distribution of the process. Due to its description complexity, we give it in three pieces, in the following theorems. Special notation local to this section is given below.

The first theorem generalises known statements for the classical random waypoint (Example A) [25], [24]. It relates the time average speed to the distribution of the speed selected at a waypoint, and contains an exact representation of the time stationary distribution of location.

Theorem 8: Under the time stationary distribution, conditional to phase $I(t)=\ell=(i, j, r$, move $)$ :

1) The numerical speed is independent of the path and the instantaneous location of the mobile at time $t$. Its density is

$$
f_{\ell}(v)=C_{\ell} \frac{1}{v} f_{V \mid \ell}^{0}(v)
$$

where $f_{V \mid \ell}^{0}(v)$ is the density of the numerical speed sampled at a transition instant and $C_{\ell}$ is a normalising constant. 
2) The path endpoints $(P(t)(0), P(t)(1))$ have a joint density over $\mathcal{A}_{i} \times \mathcal{A}_{j}$ given by

$$
\begin{aligned}
& d \mathbb{P}\left(P(t)(0)=m_{0}, P(t)(1)=m_{1} \mid I(t)=\ell\right) \\
& \quad=K_{i, j, r} d\left(m_{0}, m_{1}\right)
\end{aligned}
$$

where $K_{i, j, r}$ are normalising constants and $d(\cdot)$ is the distance in $\mathcal{A}$.

3) The distribution of $X(t)$, given $P(t)(0)=p$ and $P(t)(1)=n$, is uniform on the segment $[p, n]$.

Proof. Apply Theorem 7 to obtain the joint distribution of the path, location and speed $V(t)$, by noting that $V(t)=$ $d(P(t)(0), P(t)(1)) / S(t)$.

Comment 1. As we show later, there is no need to know the value of the constants $K_{i, j, r}$ to use the theorem in a simulation. ${ }^{2}$

Comment 2. The distribution of path endpoints $P(t)(0)$ and $P(t)(1)$ is not uniform, and the two endpoints are correlated (they tend to be far apart), contrary to what happens when sampled at transition instants. This was found already for Example A in [22].

Comment 3. One can use Theorem 8 to derive an explicit representation of the density of location $X(t)$ sampled at an arbitrary instant; for example [17] gives a closed form for the density Example A (random waypoint). However, the explicit formula is quite complicated, and is not helpful for perfect simulation. Indeed, we need to sample not only the location, but jointly location and trip, and this is readily done with Theorem 8, as we show next.

Comment 4. The relation between time stationary and event stationary distribution of speed is sometimes interpreted as "speed decay" since it is more likely to produce low speed values than the density $f_{\ell}^{0}(v)$. If one desires a uniform speed distribution in time average, then the density of speed at transition instants should be $f_{\ell}^{0}(v)=K_{\ell}^{\prime} v 1_{\left\{v_{\min }<v<v_{\max }\right\}}$. Note that such a speed distribution satisfies the stability condition in Section IV even if $v_{\min }=0$.

Theorem 9: Under the time stationary distribution, conditional to phase $I(t)=\ell=(i, j, r$,pause $)$ :

1) The location $X(t)$ and the time $R(t)$ until end of pause are independent.

2) $X(t)$ is uniform in $\mathcal{A}_{i}$.

3) $R(t)$ has density

$$
f_{\ell}(r)=\frac{1}{\bar{\tau}_{\ell}} \bar{F}_{S \mid \ell}^{0}(s)
$$

where $\bar{F}_{S \mid \ell}^{0}(s)=1-F_{S \mid \ell}^{0}(s)$ is the complementary distribution of pause time, given the phase is $\ell$.

Proof. Similar to (but simpler than) Theorem 8.

\footnotetext{
${ }^{2}$ However, in the special case of convex domains where $d(m, n)$ is the usual Euclidean distance, it is worth noting that there are known formulae: $K_{i, j, r}^{-1}=\operatorname{vol}\left(\mathcal{A}_{i}\right) \operatorname{vol}\left(\mathcal{A}_{j}\right) \bar{\Delta}_{i, j}$, for $r>0$, and else $K_{i, j, r}^{-1}=\operatorname{vol}\left(\mathcal{A}_{i}\right)^{2} \bar{\Delta}_{i, i}$, where $\operatorname{vol}\left(\mathcal{A}_{i}\right)$ is the area or volume of $\mathcal{A}_{i}$ (in square or cubic meters) and $\bar{\Delta}_{i, j}$ is the average distance in $\mathcal{A}$ between two points drawn uniformly in $\mathcal{A}_{i}$ and $\mathcal{A}_{j}$. For $r=0$ and $\mathcal{A}_{i}=$ a square of a size $a, K_{i, j, 0}^{-1} \approx 0.5214 a^{5}$; for a disk of radius $a, K_{i, j, 0}^{-1} \approx 0.9054 \pi^{2} a^{5}$ [12]. For an arbitrary case, it is generally not possible to obtain either $\operatorname{vol}\left(\mathcal{A}_{i}\right)$ or $\bar{\Delta}_{i, j}$ in a closed form, but $K_{i, j, r}^{-1}$ can be estimated directly by Monte Carlo simulation.
}

If $\bar{\Delta}$ is known

$$
\begin{aligned}
& q_{0}=\tau_{\text {pause }} /\left(\tau_{\text {pause }}+\omega \bar{\Delta}\right) \\
& \text { Draw } U_{1} \sim U(0,1) \\
& \text { if } U_{1} \leq q_{0} I(t)=\text { pause } \\
& \text { else } \\
& I(t)=\text { move } \\
& \quad \text { do } \\
& \quad \text { Draw } M_{0} \sim \operatorname{Unif}\left(\mathcal{A}_{1}\right), M_{1} \sim \operatorname{Unif}\left(\mathcal{A}_{1}\right) \\
& \quad \text { Draw } U_{2} \sim \operatorname{Unif}(0, \Delta) \\
& \text { until } U_{2}<d\left(M_{0}, M_{1}\right)
\end{aligned}
$$

else (i.e. $\bar{\Delta}$ is not known)

$$
\begin{aligned}
& q_{0}=\tau_{\text {pause }} /\left(\tau_{\text {pause }}+\omega \Delta\right) \\
& \text { do forever } \\
& \text { Draw } U_{1} \sim U(0,1) \\
& \text { if } U_{1} \leq q_{0} I(t)=\text { pause; leave } \\
& \text { else } \\
& \quad \operatorname{Draw} M_{0} \sim \operatorname{Unif}\left(\mathcal{A}_{1}\right), M_{1} \sim \operatorname{Unif}\left(\mathcal{A}_{1}\right) \\
& \operatorname{Draw} U_{2} \sim \operatorname{Unif}(0, \Delta) \\
& \text { if } U_{2}<d\left(M_{0}, M_{1}\right) \\
& I(t)=\text { move; leave }
\end{aligned}
$$

end do

Fig. 8. Sampling algorithm for restricted random waypoint with $L=1$, supporting both cases where the average distance between points in $\mathcal{A}_{1}$ is known or not. The general case $L>1$ is given in Appendix. $\tau_{\text {pause }}$ is the average pause time, $\bar{\Delta}$ the average distance in $\mathcal{A}$ between two points in $\mathcal{A}_{1}$, $\Delta$ an upper bound on the distance in $\mathcal{A}$ between two points in $\mathcal{A}_{1}$ and $\omega=$ $\mathbb{E}^{0}\left(1 / V_{0} \mid I_{0}=\right.$ move $)$.

We next show the time-stationary distribution for phase, but only for the special case $L=1$, i.e. one sub-domain. The general case for arbitrary $L$ bears some notational complexity and is for this reason deferred to Appendix.

Theorem 10: The time stationary distribution $\pi(\ell)$ to be in phase $\ell$ is

$$
\pi(\text { pause })=\frac{\tau_{\text {pause }}}{\tau_{\text {pause }}+\bar{\Delta} \omega}
$$

and $\pi($ move $)=1-\pi$ (pause $)$, where $\tau_{\text {pause }}$ is the average pause time, $\bar{\Delta}$ the average distance in $\mathcal{A}$ between two points in $\mathcal{A}_{1}$, and

$$
\omega=\mathbb{E}^{0}\left(\frac{1}{V_{0}} \mid I_{0}=\text { move }\right)
$$

is the event average of the inverse of the speed.

As with Theorem 8, we show later that we do not need to know $\bar{\Delta}$ to use this theorem for sampling.

\section{B. Perfect Simulation Without Computing Geometric Integrals}

A straightforward application of the previous section poses the problem of how to sample $m_{0}, m_{1}$ from the density in Theorem 8. Further, in order to sample the phase in Theorem 10 one needs to compute the geometric integrals $\bar{\Delta}_{i, j}$; for simple cases ( $L=1$ and $\mathcal{A}_{1}$ is a rectangle or disk) there exist closed forms, as mentioned in Comment 1 after Theorem 8. Otherwise, one needs to compute them offline by Monte Carlo simulation. For some cases, this is time consuming (see analysis in Appendix). There is generally more efficient procedure, which avoids computing the geometric integrals when they are not known, 
as we show now. The solution of these two problems is based on the following lemma.

1) Rejection Sampling Lemma: Let $(J, Y)$ be a random vector, where $J$ is in a discrete set $\mathcal{I}$ and $Y \in \mathbb{R}^{d}$. Assume that $\mathbb{P}(J=j)=\lambda \mu(j) \omega_{j}$ and the distribution of $Y$ conditional to $J=j$ has a density $\frac{f_{j}(y)}{\omega_{j}}$ The problem is to sample from $(J, Y)$ without having to compute the normalising constants of the densities $\omega_{j}$ for all $j$.

Assume we know factorisations of the form $f_{j}(y)=$ $k_{j}(y) g_{j}(y)$ where $g_{j}(y)$ is a probability density, i.e. $\int g_{j}(y) d y=$ 1 , or in other words there is no normalising constant to compute for $g_{j}(y)$. Assume also that we know upper bounds $\kappa_{j}$ such that $0 \leq k_{j}(y) \leq \kappa_{j}$.

Lemma 7: Let $v$ be the probability on $\mathcal{I}$ defined by: if $\omega_{j}$ is known $\mathrm{v}(j)=\alpha \mu(j) \omega_{j}$ else $\mathrm{v}(j)=\alpha \mu(j) \kappa_{j}$, where $\alpha$ is a normalising constant, defined by the condition $\sum_{j} v(j)=1$. The following algorithm draws a sample from $(J, Y)$ :

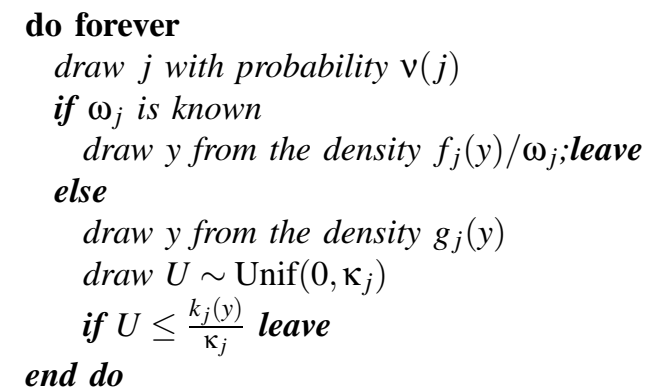

Comment. The lemma follows by the structure of the distribution of $J$ and conditional density of $Y$. The structure is: $\mathbb{P}(J=j)$ is proportional to $\omega_{j}$, while the conditional density of $Y$, given $J=j$, is inversely proportional to $\omega_{j}$. By this structure, twisting the original distribution of $J$ and conditional density of $Y$, by replacing $\omega_{j}$ with $\kappa_{j}$, indeed results in the original joint density of $(J, Y)$. The lemma is a general result. However, it may be helpful to note that the general form was suggested by particular distributions in Theorem 7. Therein, phase $I(t)$ acts the role of $J$, while $(P(t), S(t), U(t))$ acts the role of $Y$.

2) The Sampling Method: The following theorem gives the sampling method. The details for the general case have some description complexity, and is for this reason deferred to Appendix. We show all details here for the case $L=1$.

Theorem 11: (Perfect Simulation of Restricted Random Waypoint) The following algorithm draws a sample of the time stationary state of the restricted random waypoint:
1) Sample a phase $I(t)=\ell=(i, j, r, \phi)$ from the algorithm in Figure 8 (simple case) or in Appendix (general case).

2) If $\phi=$ pause

- Sample a time $\tau$ from the distribution with density $f_{\ell}(\tau)=\bar{F}_{S \mid \ell}^{0}(\tau) / \bar{\tau}_{\ell}$.

- Sample a point M uniformly in $\mathcal{A}_{i}$.

- Start the simulation in pause phase at location $M$ and schedule the end of pause at $\tau$.

3) If $\phi=$ move

- Sample a speed $v$ from the distribution with density proportional to $\frac{1}{v} f_{V \mid \ell}^{0}(v)$.

- Set $M_{0}, M_{1}$ to the value returned by the algorithm in Figure 8 (simple case) or in Appendix (general case).

- Sample u uniformly in $(0,1)$.

- Start the simulation in move phase, with initial position $(1-u) M_{0}+u M_{1}$, next trip endpoint $=M_{1}$, and speed $=v$.

Note that the algorithm in Figure 8 solves both problems mentioned in the introduction of this section.

If $\bar{\Delta}$ is known with little computational cost (i.e. when $\mathcal{A}$ is a rectangle or a disk) it is always preferable to use the former case (" $\bar{\Delta}$ is known"). Else there are two options: (i) compute $\bar{\Delta}$ offline by Monte-Carlo simulation and use the case $\bar{\Delta}$ is known", or (ii) use the case (" $\bar{\Delta}$ is not known"). Apart from unusually long simulation campaigns with the same model, the optimal choice, in terms of number of operations is to use the latter case (see Appendix). Furthermore, using the latter case simplifies the overall simulation code development. Figure 9 illustrates the sampling method on some examples from Part I.

Proof. First note (Theorem 8) that we need only to consider path and location. Then apply Theorems 8, 9 and 10. When $\bar{\Delta}_{i, j}$ is known, we solve the first problem of sampling $m_{0}, m_{1}$ from the density in Theorem 8 by applying Lemma 7 with $\mathcal{I}=\{1\}, y=\left(m_{0}, m_{1}\right), \omega_{1}=\bar{\Delta}_{i, j}, f_{1}\left(m_{0}, m_{1}\right)=$ $d\left(m_{0}, m_{1}\right) \operatorname{Unif}_{\mathcal{A}_{i}}\left(m_{0}\right) \operatorname{Unif}_{\mathcal{A}_{j}}\left(m_{1}\right), \kappa_{1}=\Delta_{i, j}$. The second problem $\left(\bar{\Delta}_{i, j}\right.$ not known) is solved by setting $\mathcal{J}=I$ and $\omega_{\ell}=\bar{\tau}_{\ell}$.

\section{Application to Examples E to G}

These are the examples where the distribution of location at an arbitrary point in time is uniform. In all of this section, we assume that the condition for existence of the time-stationary distribution (Theorem 6) is satisfied.

\section{A. Random Waypoint on Sphere}

This model is a special case of restricted random waypoint over a non convex area, with $L=1$ and $\mathcal{A}_{1}=\mathcal{A}$. Thus all findings of Section VI apply, in particular, the time stationary speed is independent of location and is given by Theorem 8 .

Theorem 12: For the random waypoint on the sphere, the time stationary distribution of the mobile location is uniform.

Proof. Apply Theorem 8. The distribution of $X(t)$ is invariant under any rotation of the sphere around an axis that 

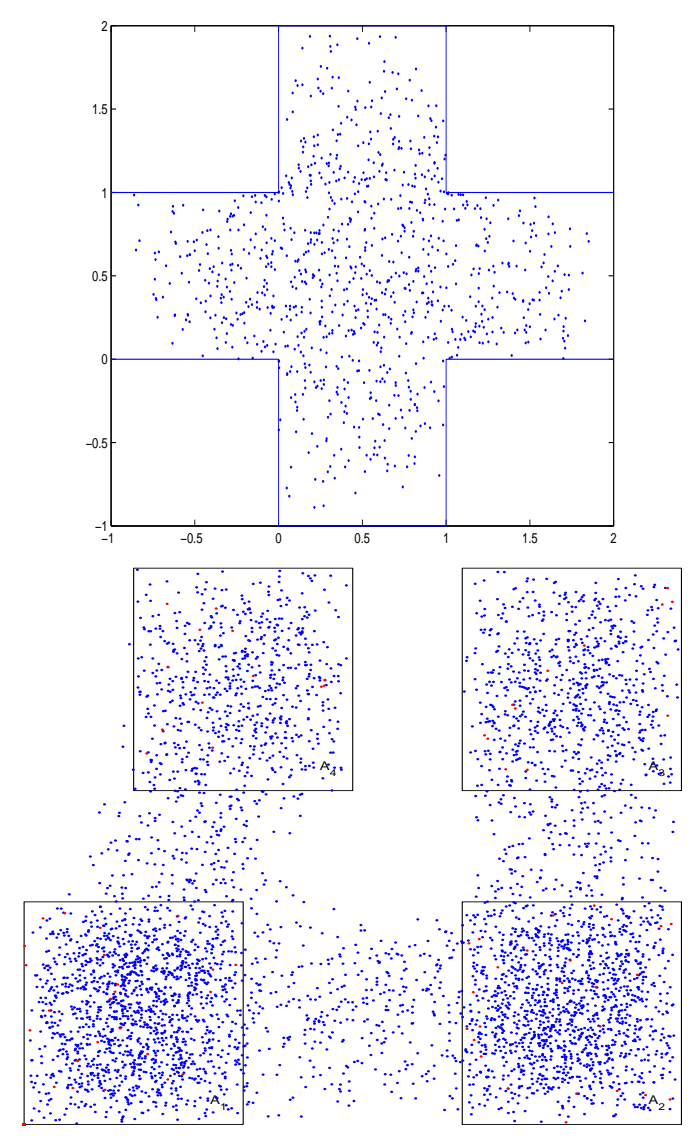
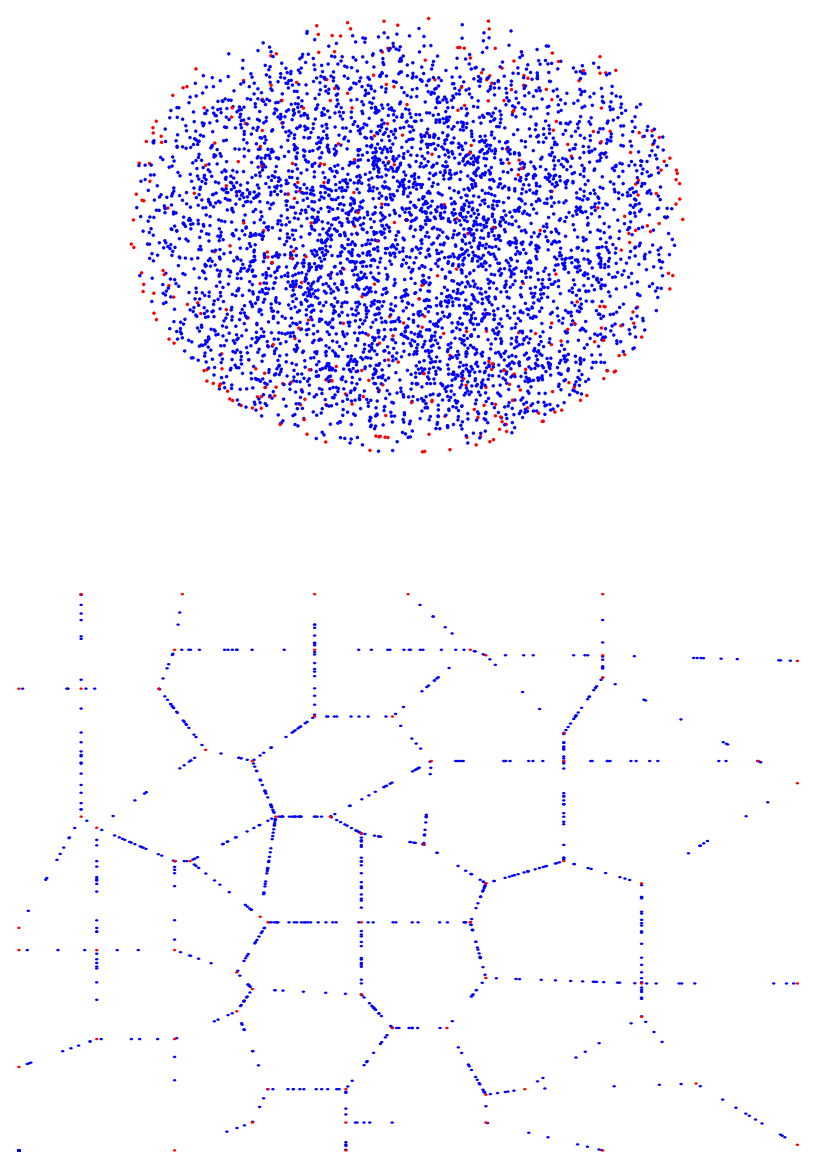

Fig. 9. Perfect sampling of node position from time-stationary distribution for examples introduced in Section III: Swiss Flag (1000 samples), Fish in a Bowl (5000 samples), Four Town restricted random waypoint (5000 samples) and Space Graph (10000 samples). Densities are not uniform, with bias towards central areas and interior corner points.

contains the centre of the sphere, and any distribution that has such an invariance property must be uniform.

Note that, with the same argument, we can show that, given we are in a move phase, the time stationary distribution of each path endpoint (previous and next) separately is also uniform, but the two endpoints are correlated (it is more likely that they are far apart). This is because, from Theorem 8, a typical path seen in time average is drawn with a probability proportional to its length. This implies that, though the time stationary distribution of points is uniform, it is not sufficient for perfect simulation to draw an initial position uniformly on the sphere and start as if it would be a path endpoint (we need in addition to sample a path and where on path according to Theorem 8).

\section{B. Random Walk on Torus}

Let $F_{\text {pause }}^{0}(t)$ [resp. $\left.F_{\text {move }}^{0}(t)\right]$ be the distribution of the pause [resp. move] duration, sampled at a transition time. Both distributions are model parameters. Also let $\bar{\tau}_{\text {pause }}, \bar{\tau}_{\text {move }}$ be the corresponding expected values (thus for example $\bar{\tau}_{\text {pause }}=$ $\mathbb{E}^{0}\left(S_{0} \mid I_{0}=\right.$ pause $\left.)=\int_{0}^{\infty} t F_{\text {pause }}^{0}(d t)\right)$. Finally, let $f_{\vec{V}}^{0}(\vec{v})$ be the density of the distribution of the speed vector (sampled at trip endpoints).
Theorem 13: For the random walk on torus, under the time stationary distribution:

1) The process state at time $t$ is fully described by the phase $I(t)$, the location $X(t)$, the speed vector $\vec{V}(t) \quad(=\overrightarrow{0}$ if $I(t)=$ pause) and the residual time until end of trip $R(t)$.

2) The location $X(t)$ is uniformly distributed.

3) $\operatorname{IP}(I(t)=$ pause $)=1-\mathbb{P}(I(t)=$ move $)=\frac{\bar{\tau}_{\text {pause }}}{\bar{\tau}_{\text {pause }}+\bar{\tau}_{\text {move }}}$.

4) Conditional $I(t)=$ pause:

- The residual pause duration $R(t)$ has density $f_{\text {pause }}(r)=\bar{F}_{\text {pause }}^{0}(s) / \bar{\tau}_{\text {pause }}$;

- $X(t)$ and $R(t)$ are independent.

5) Conditional to $I(t)=$ move:

- $\vec{V}(t)$ has density $f_{\vec{V}}^{0}(\vec{v})$;

- The residual trip duration $R(t)$ has density $f_{\text {move }}(r)=\bar{F}_{\text {move }}^{0}(s) / \bar{\tau}_{\text {move }}$;

- $X(t), \vec{V}(t)$ and $R(t)$ are independent.

Lemma 8: Let $X$ be a random point, uniformly distributed in $\mathcal{A}=\left[0, a_{1}\right] \times\left[0, a_{2}\right] \times \ldots\left[0, a_{d}\right]$. For any non random vector $\vec{v} \in \mathbb{R}^{d}$, the distribution of $w(X+\vec{v})$ is also uniform in $\mathcal{A}$.

Perfect Simulation of the random walk on torus. It follows immediately and, contrary to random waypoint on sphere, it is very simple. Pick a phase in proportion to the average time spent in the phase. Pick a point and, for the 
move phase, a speed vector as if at a transition point, and pick a remaining trip duration according to the general formula for the density of the residual time until next transition, in any stationary system. Also, there is no speed decay [26] as with random waypoint on a sphere.

\section{Billiards}

There is a similar result for the billiards, but its proof if more elaborate. We assume that the speed vector has a completely symmetric distribution, as defined in Section III-G of Part I (i.e. there is equal probability of going left or right [resp. up or down]). We continue with the same notation, in particular, the state of the simulation at time $t$ is given by the phase $I(t)$, the location $X(t)$, the speed vector $\vec{V}(t) \quad(=\overrightarrow{0}$ if $I(t)=$ pause $)$ and the residual time until end of trip $R(t)$.

Note that now there is a difference. The instant speed $\vec{V}(t)$ is, in general, not constant during an entire trip and may differ from the unreflected speed $\vec{W}_{n}$ chosen at the beginning of the trip (as it gets reflected at the boundary of $\mathcal{A}$ ). Let $f_{\vec{W}}^{0}(\vec{w})$ be the density of the distribution of the non reflected speed vector (sampled at trip endpoints).

Theorem 14: For the random walk with reflection, the same holds as in Theorem 13 after replacing the first bullet of item 5 by

- $\vec{V}(t)$ has density $f_{\vec{W}}^{0}(\vec{v})$.

The following lemma is used in the proof of Theorem 14; it says that, at the end of a trip that starts from a uniform point $M$ and a completely symmetric initial speed vector $\vec{W}$, the reflected destination point $M^{\prime}$ and speed vector $\vec{W}^{\prime}$ are independent and have same distribution as initially.

Lemma 9: Let $M$ be a random point, uniformly distributed in $\mathcal{A}$. Let $\vec{W}$ be a random vector in $\mathbb{R}^{2}$ independent of $M$ and with completely symmetric distribution. Let $\alpha \in \mathbb{R}$ be a constant. Define $M^{\prime}=b(M+\alpha \vec{W})$ and $\vec{W}^{\prime}=J_{M+\alpha \vec{W}}(\vec{W})$. $\left(M^{\prime}, \vec{W}^{\prime}\right)$ has the same joint distribution as $(M, \vec{W})$.

Remark. It is important to use the instant speed vector $\vec{V}(t)$ and not the unreflected speed vector $\vec{W}(t)$ when describing the simulation state: indeed the description by phase $I(t)$, location $X(t)$, unreflected speed vector $\vec{W}(t) \quad(=\overrightarrow{0}$ if $I(t)=$ pause $)$ and residual time until end of trip $R(t)$ is not sufficient to continue the simulation (one needs to remember which reflection was applied to the speed vector) and is thus not Markov.

Also note that, in time stationary averages, the location $X(t)$ and the unreflected speed vector $\vec{W}(t)$ are not independent. For example, given that the unreflected speed vector is $\vec{W}(t)=$ $\left(0.5 a_{1}, 0\right)$ and the trip duration is $S(t)=1$, it is more likely that $X(t)$ is in the second right half of the rectangle. In contrast, $X(t)$ and the instant speed vector $\vec{V}(t)$ are independent, as shown by the theorem.

Perfect simulation of the billiards. It is similar to the random walk on torus.

\section{RELATED WORK}

For a survey of existing mobility models, see the work by Camp, Boleng, and Davies [10] and the references therein.
Bettstetter, Harnstein, and Pérez-Costa [12] studied the timestationary distribution of a node location for classical randomwaypoint model. They observed that the time-stationary node location is non-uniform and it has more mass in the center of a rectangle. A similar problem has been further studied by Bettstetter, Resta, and Santi [5]. A closed-form expression for the time-stationary density of a node location is obtained only for random-waypoint on a one-dimensional interval; for two dimensions only approximations are obtained. Note that in Theorem 8, we do have an exact representation of the distribution of node location as a marginal of a distribution with a known density. Neither [12] nor [5] consider how to run perfect simulations.

It is the original finding of Yoon, Liu, and Noble [25] that the default setting of the classical random-waypoint exhibits speed decay with time. The default random-waypoint assumes the event-stationary distribution of the speed to be uniform on an interval $(0, v \max ]$. The authors found that if a node is initialized such that origin is a waypoint, the expected speed decreases with time to 0 . This in fact is fully explained by the infinite expected trip duration as sampled at trip transitions, which implies the random process of mobility state is nullrecurrent; see Section IV. In a subsequent work [26], the same authors advocate to run "sound" mobility models by initializing a simulation by drawing a sample of the speed from its time-stationary distribution. We remark that this is only a partial solution as speed is only a component of node mobility state. For this reason, the authors in [26] do not completely solve the problem of perfect simulation. Another related work is that of Lin, Noubir, and Rajaraman [18] that studies a class of mobility models where travel distance and travel speed between transition points can be modeled as a renewal process. The renewal assumption was also made in [25], [26]. We note that this assumption is not verified with mobility models such as classical random-waypoint on any non-isotropic domain, such as a rectangle, for example. The renewal assumption has been made to make use of a "cycle" formula from the theory of renewal random processes. From Palm calculus, we know that the "cycle" formula is in fact Palm inversion formula, which we used extensively throughout the paper, and that applies more generally to stationary random processes; this renders the renewal assumption unnecessary.

Perhaps the work closest to ours is that of Navidi, Camp, and Bauer in [24], [22]. As discussed in Section I-D, we provide a systematic framework that allows to formally prove some of the implicit statements in [22] and generalize to a broader class. Further, our perfect sampling algorithm differs in that it works even when geometric constants are not a priori known. In [21], Nain, Towsley, Liu and Liu consider the random walk on torus and billiards models (which they call "random direction"), assuming the speed vectors are isotropic. They find that the stationary regime has uniform distribution, and advocate that this provides an interesting bias-free model.

There are other well established techniques for performing perfect simulation. The method in [23] applies to a large class of Markov chains on which some partial ordering can be defined, and uses coupling from the past (sample trajectories starting in the past at different initial conditions). The tech- 
nique presented in this paper is much simpler, as, unlike in the case of [23], we can obtain an explicit representation of the stationary distribution.

\section{CONCLUSION}

The random trip model provides a framework to analyse and simulate stable mobility models that are guaranteed to have a unique time-stationary distribution. Moreover, conditions are provided that guarantee convergence in distribution to a timestationary distribution, from origin of an arbitrary trip. It is showed that many known random mobility models are random trip models.

For stable random trip models, if initial node mobility state is not sampled from the time-stationary distribution, the node mobility state distribution converges to the time-stationary distribution. The rate of this convergence depends on the geometry of the mobility domain and specifics of the trip selection. In order to alleviate this initial transience altogether, we provide a perfect sampling algorithm to initialise node mobility state to a sample from the time-stationary distribution, so that a node movement is a time-stationary realisation.

The web page "random trip model":

- http://ica1wwww.epfl.ch/RandomTrip

provides a repository of random trip models and a free to download perfect sampling software to use in simulations.

\section{ACKNOWLEDGMENTS}

The perfect sampling ns-2 code pointed out in this paper was implemented by Santashil PalChaudhuri. M. V. is grateful to Srdjan Čapkun for referring him to the mobility problem studied in this paper and thanks Don Towsley for encouragement and discussions.

\section{REFERENCES}

[1] Gerold Alsmeyer. The markov renewal theorem and related results. Markov Proc. Rel. Fields, 3:103-127, 1997.

[2] Soren Asmussen. Applied Probability and Queues. Springer, 2 edition, 2003.

[3] François Baccelli and Pierre Brémaud. Palm Probabilities and Stationary Queues. Springer LNS, 1987.

[4] Francois Baccelli and Pierre Brémaud. Elements of Queueing Theory: Palm Martingale Calculus and Stochastic Recurrences, volume 26. Applications of Mathematics, Springer-Verlag, 2 edition, 2003.

[5] Christian Bettstetter, Giovanni Resta, and Paolo Santi. The node distribution of the random waypoint mobility model for wireless ad hoc networks. IEEE Transactions On Mobile Computing, 2(3), JulySeptember 2003.

[6] Ljubica Blažević, Jean-Yves Le Boudec, and Silvia Giordano. A location based routing method for mobile ad hoc networks. IEEE Transactions on Mobile Computing, 3(4), December 2004

[7] Jean-Yves Le Boudec and Milan Vojnović. Perfect Simulation and Stationarity of a Class of Mobility Models. In Proceedings of IEEE Infocom 2005, Miami, Florida, USA, March 2005.

[8] Pierre Brémaud. Markov Chains: Gibbs Fields, Monte Carlo Simulation and Queues. Springer, 1999.

[9] Josh Broch, David A. Maltz, David B. Johnson, Yih-Chun Hu, and Jorjeta Jetcheva. A performance comparison of multi-hop wireless ad hoc network routing protocols. In Mobile Computing and Networking, pages 85-97, 1998.

[10] T Camp, J. Boleng, and V. Davies. A survey of mobility models for ad hoc network research. WCMC: Special issue on Mobile Ad Hoc Networking: Research, Trends and Applications, 2(5):483-502, 2002.

[11] Erhan Çinlar. Introduction to Stochastic Processes. Prentice Hall, 1975.
[12] Hannes Hartenstein Christian Bettstetter and Xavier Pérez-Costa Stochastic properties of the random waypoint mobility model ACM/Kluwer Wireless Networks, Special Issue on Modeling and Analysis of Mobile Networks 2003.

[13] G. Hardy, J. E. Littlewood, and G. Pólya. Inequalities. Cambridge University Press, 2 edition, 1952.

[14] Amit Jardosh, Elizabeth M. Belding-Royer, Kevin C. Almeroth, and Subhash Suri. Towards realistic mobility models for mobile ad-hoc networking. In Proc. of ACM Mobicom 2003, pages 217-229, San Diego, CA, 2003

[15] B. M. Kloss. Probability Distributions on Bicompact Topological Groups. Theory of Probability and its Application, IV:237-270, 1959.

[16] Jean-Yves Le Boudec. On the stationary distribution of speed and location of random waypoint. IEEE Transactions on mobile computing, 4(4):404-405, 2005

[17] Jean-Yves Le Boudec. Understanding the simulation of mobility models with palm calculus. Performance Evaluation, to appear, also available as EPFL Technical Report EPFL/IC/2004/53.

[18] Guolong Lin, Guevara Noubir, and Rajmohan Rajamaran. Mobility models for ad-hoc network simulation. In Proceedings of Infocom 2004, April 2004.

[19] Torgny Lindvall. Lectures on the Coupling Method. Dover (originally published by John Wiley \& Sons in 1992), 2002.

[20] M.Drmota and R. F. Tichy. Sequences, Discrepancies and Applications. Lecture Notes in Math. 1651, Springer, 1997.

[21] Philippe Nain, Don Towsley, Benyuan Liu, and Zhen Liu. Properties of Random Direction Models. In Proceedings of IEEE Infocom 2005 , Miami, Florida, USA, March 2005.

[22] William Navidi and Tracy Camp. Stationary distributions for the random waypoint model. IEEE Transactions on Mobile Computing, 3(1), 2004.

[23] James G. Propp and David B. Wilson. Exact sampling with coupled markov chains and applications to statistical mechanics. Random Structures and Algorithms, 9(1 \& 2):223-252, 1996.

[24] T. Camp W. Navidi and N. Bauer. Improving the accuracy of random waypoint simulations through steady-state initialization. In Proceedings of the 15th International Conference on Modeling and Simulation (MS '04), pages 319-326, March 2004.

[25] Jungkeun Yoon, Mingyan Liu, and Brian Noble. Random waypoint considered harmful. In Proceedings of Infocom, 2003.

[26] Jungkeun Yoon, Mingyan Liu, and Brian Noble. Sound mobility models. In Proceedings of Mobicom, 2003.

\section{APPENDIX}

\section{A. Proof of Theorem 3}

By Theorem 2, it suffices to consider a model with no pauses. $I_{n} \equiv\left(K_{n}, L_{n}, R_{n}\right)$ is indeed a Markov chain on a countable state space given by $I=\bigcup_{(i, j) \in \mathcal{L}_{2}}\{i\} \times\{j\} \times \mathcal{R}$, with $\mathcal{L}_{2}=\left\{(i, j) \in \mathcal{L}^{2}: Q(i, j)>0\right\}$. Here $\mathcal{R}_{i}=\mathbb{Z}_{+}$, if there exists no finite $r_{i}$ such that $F_{i}\left(r_{i}\right)=1$, else $\mathcal{R}_{i}=\left\{0,1, \ldots, r_{i}\right\}$. Conditional on all observed past for transitions $k \leq n$, including phase $I_{n}$ and path $P_{n}=\left(M_{n}, M_{n+1}\right)$, the distribution of $\left(I_{n+1}, P_{n+1}\right)$, depends only on $\left(I_{n}, P_{n}\right)$, thus $Y_{n}=\left(I_{n}, P_{n}\right)$ is a Markov chain. This shows that $\mathrm{H} 1$ holds. Condition H4 indeed holds by the model definition. We next show that $\mathrm{H} 2$ holds. Note that the prevailing model permits us to verify $\mathrm{H} 2$ by essentially considering only the Markov chain $I_{n}$, which takes values on a countable state space, and thus standard stability results can be employed.

Condition H2.i holds for a recurrent set $R=(i, j, r) \times \mathcal{A}^{2}$, where $(i, j, r)$ is any fixed element of $I$. Indeed, first note that $I_{n}$ is an irreducible Markov chain, which follows from the assumed irreducibility of the transition matrix $Q$ that specifies the Markov walk on the subdomains. Define the function $V$ : $I \rightarrow \mathbb{R}$ as $V((i, j, r))=r$, for $(i, j, r) \in I$ and let $H$ be a set given by $H:=\mathcal{L}_{2} \times\{0\}$. It holds that for any $(i, j, 0) \in H$

$$
\mathbb{E}\left(V\left(I_{1}\right) \mid I_{0}=(i, j, 0)\right)=\mathbb{E}\left(R_{1} \mid I_{0}=j\right)<+\infty,
$$


and, for any $(i, j, r) \notin H$,

$$
\mathbb{E}\left(V\left(I_{1}\right) \mid I_{0}=(i, j, r)\right)-V(i, j, r)=-1<0 .
$$

The former relation is by hypothesis of the theorem and latter by the fact that $R_{n+1}=R_{n}-1$, whenever $R_{n}>0$. By Foster's theorem [8, Theorem 1.1-Chapter 5], this implies $I_{n}$ is a positive recurrent chain and thus H2.i is true by taking any subset of $I$ as a recurrent set.

We next show that H2.ii holds as well. First, we assume that $\mathcal{R}_{i}$ is finite for all $i \in \mathcal{L}$ and then later remove this assumption. Note that for any $n \geq 2$ and any $y \in I \times \mathcal{P}$

$$
\begin{array}{cl}
\mathbb{P}_{y}\left(I_{n}=(i, j, r), P_{n} \in A_{1} \times A_{2}\right) & \\
= & \begin{cases}\operatorname{Unif}_{i}\left(A_{1}\right) \operatorname{Unif}_{j}\left(A_{2}\right) \mathbb{P}_{y}\left(I_{n}=(i, j, 0)\right) & r=0, \\
\operatorname{Unif}_{i}\left(A_{1}\right) \operatorname{Unif}_{i}\left(A_{2}\right) \mathbb{P}_{y}\left(I_{n}=(i, j, r)\right) & r>0 .\end{cases}
\end{array}
$$

where $\operatorname{Unif}_{i}(\cdot)$ is uniform distribution on a subdomain $i \in \mathcal{L}$. It follows that it is sufficient to verify $\mathrm{H} 2$.ii for $I_{n}$. We already noted that $I_{n}$ is positive recurrent and thus has a unique invariant probability distribution $\pi_{I}^{0}$. Assume first that $I_{n}$ is aperiodic. The Markov chain $I_{n}$ is ergodic and thus the following result holds [8, Theorem 2.1-Chapter 4]:

$$
\lim _{n \rightarrow+\infty} \sum_{v \in I}\left|p_{u v}(n)-\pi_{I}^{0}(v)\right|=0, \quad \text { all } u \in I,
$$

where $p_{u v}(n)$ is the probability of the transition from a state $u$ to a state $v$ in $n$ transitions. This implies that for any $\varepsilon>0$ there exists $n_{0} \geq 1$ such that for all $n \geq n_{0}$,

$$
p_{u v}(n) \geq \pi_{I}^{0}(v)-\varepsilon \text {. }
$$

It thus follows that in $\mathrm{H} 2 . \mathrm{ii}$ we can define the probability measure $\varphi(B)=\left(\pi_{I}^{0}(B)-\varepsilon\right) /(1-\varepsilon), B \in I$, and $\beta=1-\varepsilon$ with any fixed $\varepsilon \in\left(\min _{e \in I} \pi_{I}^{0}(e), 1\right)$. Finally, assume $I_{n}$ is periodic. As all the states in $I$ communicate, $I_{n}$ is periodic with a period $d>1$ common to all the states. Define the regeneration set to be a cycle class $R$ of $I$. Then, H2.ii follows similarly as in the aperiodic case, but using instead this convergence result [8, Theorem 2.3-Chapter 4]:

$$
\lim _{n \rightarrow+\infty} \sum_{i \in R}\left|p_{u v}(n d)-d \pi_{I}^{0}(v)\right|=0, \quad \text { all } u \in R .
$$

This allows us to identify a probability measure $\varphi(\cdot)$ in H2.ii that puts all mass on $R$, i.e. $\varphi(R)=1$. As an aside remark, note that we already chosen a regeneration set as a cycle class for a special periodic Markov chain of phases of classical random waypoint with pauses in the proof of Theorem 2 .

To complete the proof that H2.ii is verified, it is left to consider the case: $\mathcal{R}_{i}=\mathbb{Z}_{+}$, for some $i \in \mathcal{L}$. This case is considered separately as in the case when $I$ is countable and infinite, $\min _{e \in I} \pi_{I}^{0}(e)=0$, the above proof cannot be taken verbatim, but with only a few slight modifications. The little technical difficulty is resolved next for $I_{n}$ aperiodic; it follows similarly for the periodic case. We let $\mathcal{J}=\{(i, j, r) \in I: r \leq$ $\left.r_{0}\right\}$, for a finite integer $r_{0} \geq 0$ such that $\min _{e \in \mathcal{J}} \pi_{I}^{0}(e)>0$. Then, define $\varphi(B)=\left(\pi_{I}^{0}(B)-\varepsilon\right) /\left(\pi_{I}^{0}(\mathcal{I})-\varepsilon\right)$ for $B \in \mathcal{J}$ and $\varphi(B)=0$, for $B \in I / \mathcal{I}$, and $\beta=\pi_{I}^{0}(\mathcal{I})-\varepsilon$. We can now indeed choose $\varepsilon \in\left(\min _{e \in \mathcal{J}} \pi_{I}^{0}(e), \pi_{I}^{0}(\mathcal{J})\right)$.

Condition H3 indeed holds for a recurrent set $R=\{e\} \times \mathcal{A}^{2}$, $e \in I$, by positive recurrence of the chain $I_{n}$.

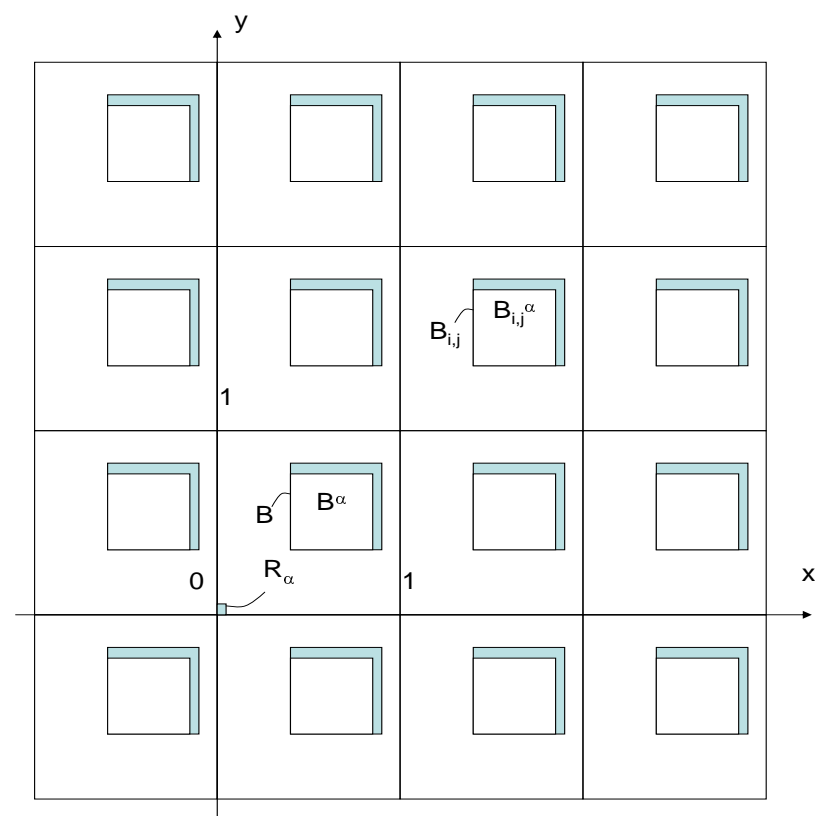

Fig. 10. The tessellation used for random walk on torus. $R_{\alpha}=[0, \alpha] \times[0, \alpha]$ is the chosen recurrent set. The bounding rectangles are the sets $B_{i j}$, the interior ones (i.e. minus the shaded areas) are $B_{i j}^{\alpha}$.

\section{B. Proof of Theorem 4}

$\mathrm{H} 1$ and $\mathrm{H} 4$ are obviously satisfied (the non lattice condition H4.iii follows from the assumption that either the distribution of the trip durations or distribution of pause times have a density). To show $\mathrm{H} 2$, by Theorem 2 we can restrict to the random walk without pauses.

In this case we have $Y_{n}=\left(M_{n}, \vec{V}_{n}, S_{n}\right)$. We can use Lemma 1. Let $R_{0}$ be a recurrent set for the chain $M_{n}$, and let $R=R_{0} \times \mathbb{R}_{+}^{3}$. H2.i holds because

$$
\mathbb{P}_{y}\left(Y_{n} \in R\right)=\mathbb{P}_{y}\left(M_{n} \in R_{0}\right)=\mathbb{P}\left(M_{n} \in R_{0} \mid M_{0}=m\right)=1
$$

where $y=(m, \vec{v}, s)$.

Since $M_{n}$ is Harris recurrent there exists some positive integer $n_{0}, \beta_{0} \in(0,1)$ and a probability $\phi_{0}$ on $\mathcal{A}$ such that for any measurable subset of $\mathcal{A}$ and any initial position $m \in \mathcal{A}$ :

$$
\mathbb{P}\left(M_{n_{0}} \in B \mid M_{0}=m\right) \geq \beta_{0} \phi_{0}(B) .
$$

Let $f_{\vec{V}}^{0}(v)$ be the density of the speed vector and $F_{S}^{0}(s)$ distribution of the trip duration. Since $S_{n}$ and $\vec{V}_{n}$ are drawn independently of the past and $M_{n}$, we have, for any $y \in R$ :

$$
\begin{aligned}
& \mathbb{P}_{y}\left(\left(M_{n_{0}} \in B, \vec{V}_{n_{0}} \in B_{1}, S_{n_{0}} \in B_{2}\right)\right. \\
= & \mathbb{P}\left(M_{n_{0}} \in B \mid M_{0}=m\right) \psi_{1}\left(B_{1}\right) \psi_{2}\left(B_{2}\right) \\
\geq & \beta_{0} \phi(B) \psi_{1}\left(B_{1}\right) \psi_{2}\left(B_{2}\right)
\end{aligned}
$$

where $\psi\left(B_{1}\right)=\int_{B_{1}} f_{\vec{V}}^{0}(v) d v$ and $\psi_{2}\left(B_{2}\right)=\int_{B_{2}} F_{S}^{0}(d s)$. This shows that $\mathrm{H} 2$.ii holds for any measurable set of the form $B \times B_{1} \times B_{2}$. It follows that $\mathrm{H} 2$.ii is also true for any union of disjoint sets of this form, and thus for any measurable set.

\section{Proof of Lemma 1}

We give the proof for $a_{1}=a_{2}=1$. The sequence $M_{n}$ is a random walk on the torus, which can be viewed as the set 
$[0,1) \times[0,1)$ endowed with componentwise addition modulo 1. This is a compact group, and, in general, a random walk on a compact group converges to a uniform distribution. More specifically, the assumption that the speed vector has a density implies that $b\left(\vec{V}_{n}\right)$ also has one with respect to the uniform measure on the torus, and by [15, Section 5.2], this implies that the distribution of $M_{n}$ converges weakly to uniform distribution on the torus.

We now show Harris recurrence, i.e. that conditions H2.i and $\mathrm{H} 2$.ii hold for the chain $M_{n}$. We take as recurrence set a small neighbourhood of the origin $R_{\alpha}:=[0, \alpha] \times[0, \alpha]$, where $\alpha$ will be fixed later.

We first show that H2.i holds for any choice of $\alpha \in(0,1)$. By the uniform convergence (Lemma 2), we have that for any $\varepsilon>0$, there exists $d \geq 1$ such that

$$
\mathbb{P}_{m}\left(M_{n} \in B\right) \leq \operatorname{Unif}(B)+\varepsilon, \text { all } n \geq d,
$$

for all product of intervals $B$ in $\mathcal{A}$ and all $m \in \mathcal{A}$. Now, fix $\varepsilon \in\left(0, \alpha^{2}\right)$ and $d$ such that (8) holds. Consider the sampled chain $M_{n d}, n=0,1, \ldots$ Note

$$
\begin{aligned}
& \mathbb{P}_{m}\left(M_{2 d} \notin R_{\alpha}, M_{d} \notin R_{\alpha}\right) \\
= & \int_{\mathscr{A} \backslash R_{\alpha}} \mathbb{P}_{y}\left(M_{d} \notin R_{\alpha}\right) \mathbb{P}_{m}\left(M_{d} \in d y\right) \\
\leq & \left(\operatorname{Unif}\left(\mathcal{A} \backslash R_{\alpha}\right)+\varepsilon\right) \mathbb{P}_{m}\left(M_{d} \notin R_{\alpha}\right) \\
\leq & \left(\operatorname{Unif}\left(\mathcal{A} \backslash R_{\alpha}\right)+\varepsilon\right)^{2} \\
\leq & \left(1-\alpha^{2}+\varepsilon\right)^{2} .
\end{aligned}
$$

Following the same argument, we have

$$
\mathbb{P}_{m}\left(\cap_{n=1}^{k}\left(M_{n d} \notin R_{\alpha}\right)\right) \leq\left(1-\alpha^{2}+\varepsilon\right)^{k} .
$$

Now, the left-hand side of $\mathrm{H} 2 . \mathrm{i}$ reads as

$$
\begin{aligned}
\mathbb{P}_{m}\left(\cup_{n=1}^{\infty} M_{n} \in R\right) & =1-\lim _{k \rightarrow+\infty} \mathbb{P}\left(\cap_{n=1}^{k}\left(M_{n} \notin R_{\alpha}\right)\right) \\
& \geq 1-\lim _{k \rightarrow+\infty} \mathbb{P}\left(\cap_{n=1}^{k}\left(M_{n d} \notin R_{\alpha}\right)\right) \\
& \geq 1-\lim _{k \rightarrow+\infty}\left(1-\alpha^{2}+\varepsilon\right)^{k} \\
& =1
\end{aligned}
$$

where we used (9) and the fact that we chosen $\varepsilon>0$ such that $1-\alpha^{2}+\varepsilon<1$. As we arbitrarily fixed $\alpha \in(0,1)$, this shows that $\mathrm{H} 2 . \mathrm{i}$ holds for any $\alpha \in(0,1)$.

We next show that $\mathrm{H} 2$.ii holds for $n_{0}=1$ and for some appropriate choice of $\alpha \in(0,1)$. It is sufficient to prove H2.ii for $B$ equal to a box of the form $\left[x_{1}, y_{1}\right] \times\left[x_{2}, y_{2}\right]$, (with $x_{1}<y_{1}$ and $\left.x_{2}<y_{2}\right)$ since any measurable set can be approximated, up to a 0 measure set, by a disjoint union of such boxes.

Define $B^{\alpha}$ as the set derived from $B$ by removal of an upper and right band of width $\alpha$ :

$$
B^{\alpha}=B \cap\left(\left[0, y_{1}-\alpha\right] \times\left[0, y_{2}-\alpha\right]\right) .
$$

Note that $B^{\alpha}$ is non empty if $\alpha<\left|y_{1}-x_{1}\right|$ or $\alpha<\left|y_{2}-x_{2}\right|$.

Also define, for $i, j \in \mathbb{Z}: B_{i, j}=B+(i, j)$ and $B_{i, j}^{\alpha}=B^{\alpha}+(i, j)$ (Figure 10). We have

$$
\begin{aligned}
\mathbb{P}_{m}\left(M_{1} \in B\right) & =\mathbb{P}_{m}\left(w\left(m+\vec{V}_{1}\right) \in B\right) \\
& =\mathbb{P}_{m}\left(m+\vec{V}_{1} \in \cup_{i, j} B_{i, j}\right) \\
& =\sum_{i, j} \mathbb{P}_{m}\left(m+\vec{V}_{1} \in B_{i j}\right) \\
& \geq \sum_{i, j} \mathbb{P}_{0}\left(\vec{V}_{1} \in B_{i j}^{\alpha}\right) \\
& =\mathbb{P}_{0}\left(\vec{V}_{1} \in \cup_{i, j} B_{i j}^{\alpha}\right):=g_{\alpha}(B) .
\end{aligned}
$$

Thus, in H2.ii, we can set $\beta=g_{\alpha}([0,1) \times[0,1))$ and $\varphi_{\alpha}(B)=$ $g_{\alpha}(B) / \beta$, provided that $g_{\alpha}([0,1) \times[0,1))>0$. But this indeed holds for some $\alpha \in(0,1)$ as follows from $\lim _{n \rightarrow+\infty} g_{\alpha_{n}}([0,1) \times$ $[0,1))=1$, where $\alpha_{n}$ is any sequence decreasing to 0 .

We are left only to verify the condition H3. But this follows from (9) that says the number of transitions between successive visits to the recurrent set $R_{\alpha}$ is stochastically smaller than (finite integer) $d$ times a geometric random variable with a fixed parameter in $(0,1)$.

\section{Proof of Lemma 2}

We use Erdös-Turán-Koksma inequality [20, Theorem 1.21], which says that for any positive integer $H$, any probability distribution $\mu$ on the torus, and any product of intervals $B$ we have

$$
\begin{aligned}
& |\mu(B)-\operatorname{Unif}(B)| \\
& \leq\left(\frac{3}{2}\right)^{2}\left(\frac{2}{H+1}+\sum_{0<\max \left(h_{1}, h_{2}\right) \leq H} \frac{\left|\hat{\mu}\left(h_{1}, h_{2}\right)\right|}{R\left(h_{1}, h_{2}\right)}\right)
\end{aligned}
$$

where $h_{1}, h_{2}$ are integers, $R\left(h_{1}, h_{2}\right)=\max \left(1, h_{1}\right) \max \left(1, h_{2}\right)$ and $\hat{\mu}\left(h_{1}, h_{2}\right)$ are the Fourier coefficients of $\mu$ :

$$
\hat{\mu}\left(h_{1}, h_{2}\right):=\int_{[0,1) \times[0,1)} e^{-2 i \pi\left(h_{1} x_{1}+h_{2} x_{2}\right)} d \mu\left(x_{1}, x_{2}\right) .
$$

We apply Equation (10) to $\mu=\mu_{m}^{* n}$ and obtain

$$
\begin{aligned}
e^{-2 i \pi\left\langle\left(h_{1}, h_{2}\right), M_{n}\right\rangle} & =e^{-2 i \pi\left\langle\left(h_{1}, h_{2}\right), b\left(M_{0}+\sum_{j=0}^{n-1} \vec{V}_{j}\right)\right\rangle} \\
& =e^{-2 i \pi\left\langle\left(h_{1}, h_{2}\right), M_{0}+\sum_{j=0}^{n-1} \vec{V}_{j}\right\rangle}
\end{aligned}
$$

where the last equality is because $h_{1}, h_{2}$ are integers $(\langle\cdot, \cdot\rangle$ is the scalar product). Thus

$$
\begin{aligned}
\hat{\mu}_{m}^{* n}\left(h_{1}, h_{2}\right) & :=\mathbb{E}_{m}\left(e^{-2 i \pi\left\langle\left(h_{1}, h_{2}\right), M_{n}\right\rangle}\right) \\
& =e^{-2 i \pi\left\langle\left(h_{1}, h_{2}\right), m\right\rangle} \mathbb{E}_{m}\left(\Pi_{j=0}^{n-1} e^{-2 i \pi\left\langle\left(h_{1}, h_{2}\right), \vec{V}_{j}\right\rangle}\right) \\
& =e^{-2 i \pi\left\langle\left(h_{1}, h_{2}\right), m\right\rangle} \Pi_{j=0}^{n-1} \mathbb{E}_{m}\left(e^{-2 i \pi\left\langle\left(h_{1}, h_{2}\right), \vec{V}_{j}\right\rangle}\right)
\end{aligned}
$$

where $\mathbb{E}_{m}$ denotes the conditional expectation given that $M_{0}=m$. Thus

$$
\left|\hat{\mu}_{m}^{* n}\left(h_{1}, h_{2}\right)\right| \leq\left|\hat{f}_{\vec{V}}\left(h_{1}, h_{2}\right)\right|^{n}
$$

where $\hat{f}_{\vec{V}}\left(h_{1}, h_{2}\right)$ are the Fourier coefficients of the distribution of the speed vector.

Now $\vec{V}_{n}$ has a density thus if $\left(h_{1}, h_{2}\right) \neq(0,0)$, the scalar product $\left\langle\left(h_{1}, h_{2}\right), \vec{V}_{n}\right\rangle$ also has a density, and is thus nonlattice. By Lemma 3:

$$
\left|\hat{f}_{\vec{V}}\left(h_{1}, h_{2}\right)\right|<1 \text { for }\left(h_{1}, h_{2}\right) \neq(0,0) .
$$




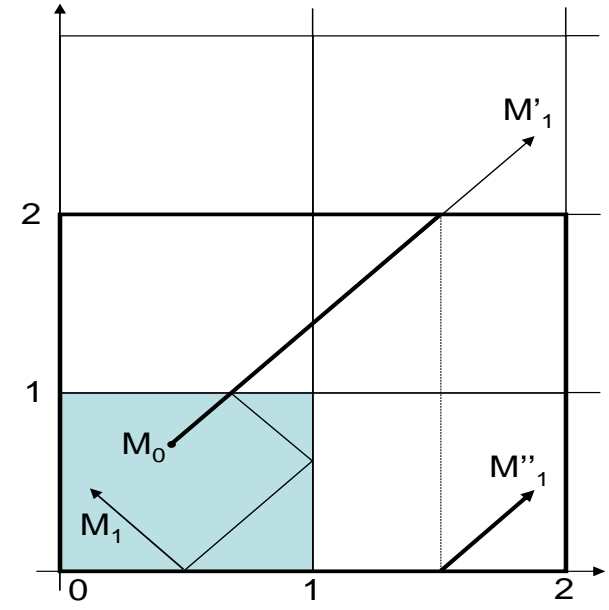

Fig. 11. Random walk $M_{n}^{\prime \prime}$ on $[0,2) \times[0,2)$ associated with billiards $M_{n}$ on $[0,1) \times[0,1)$.

We can now use Equations (10), (12) and (13) to Equation (4). Indeed, fix some arbitrary $\varepsilon>0$, and fix some $H$ such that $\left(\frac{3}{2}\right)^{2} \frac{2}{H+1}<\varepsilon / 2$. The summation in Equation (10) is finite, thus by Equation (13) it goes to 0 as $n$ grows to infinity. Thus, for $n$ large enough, it is smaller than $\varepsilon / 2$. This shows that there is some $n_{1}$ (independent of $m$ and $I$ ) such that for $n \geq n_{1}$, the right-hand side in Equation (10) is less than $\varepsilon$.

\section{E. Proof of Lemma 4}

It suffices to consider $a_{1}=a_{2}=1$. The lemma relies on a reduction to a random walk, with wrapping. First define, for any location $\left(x_{0}, y_{0}\right) \in \mathbb{R}^{2}$ the (linear) operator $J_{\left(x_{0}, y_{0}\right)}$ by

$$
J_{\left(x_{0}, y_{0}\right)}(x, y)=\left((-1)^{\left\lfloor x_{0}\right\rfloor} x,(-1)^{\left\lfloor y_{0}\right\rfloor} y\right) .
$$

The linear operator $J_{m}$ expresses exactly the transformation on the unwrapped speed vector (Figure 7), i.e.

$$
\vec{V}_{n+1}=J_{M_{n}+S_{n} \vec{W}_{n}}\left(\vec{W}_{n}\right) \text {. }
$$

We now associate to the sequence $M_{n}$ two sequences $M_{n}^{\prime}$ (non reflected) defined by

$$
M_{0}^{\prime}=M_{0} \quad M_{n+1}^{\prime}=M_{n}^{\prime}+J_{M_{n}^{\prime}}\left(\vec{U}_{n}\right)
$$

and $M_{n}^{\prime \prime}$ (wrapped modulo 2) defined by

$$
M_{n}^{\prime \prime}=w_{2}\left(M_{n}^{\prime}\right)
$$

where $w_{2}(\cdot)$ is the wrapping mapping defined by (3) with $a_{1}=$ $a_{2}=2$ (Figure 11).

We now show by induction on $n \in \mathbb{N}$ that $b\left(M_{n}^{\prime}\right)=M_{n}$ for all $n$, i.e. $M_{n}^{\prime}$ is the unreflected version of $M_{n}$. This is true by definition for $n=0$. Assume it holds for $n$. By application of Lemma 5 to $m=M_{n}^{\prime}$ and $\vec{v}=J_{M_{n}^{\prime}}\left(\vec{U}_{n}\right)$ we obtain

$$
\begin{aligned}
b\left(M_{n+1}^{\prime}\right) & =b\left(M_{n}+J_{M_{n}^{\prime}}\left(J_{M_{n}^{\prime}}\left(\vec{U}_{n}\right)\right)\right) \\
& =b\left(M_{n}+\vec{U}_{n}\right)=M_{n+1}
\end{aligned}
$$

where we have used the fact that $J_{m}$ is its own inverse.
It follows now from and by Lemma 6 that

$$
b\left(M_{n}^{\prime \prime}\right)=M_{n} \quad \text { all } n \in \mathbb{Z}_{+},
$$

i.e. the billiards $M_{n}$ is derived from the wrapped sequence $M_{n}^{\prime \prime}$ by reflection.

Now we show that $M_{n}^{\prime \prime}$ is a random walk on the torus with $a_{1}=a_{2}=2$, as defined in Section III-F. It follows from Equations (15) and (16) that

$$
M_{n+1}^{\prime \prime}=M_{0} \oplus \vec{U}_{0}^{\prime \prime} \oplus \cdots \oplus \vec{U}_{n}^{\prime \prime}
$$

where here $\oplus$ is addition modulo 2 and $\vec{U}_{n}^{\prime \prime}=J_{M_{n}^{\prime}}\left(\vec{U}_{n}\right)$. In general, we do not have a random walk (with independent increments) due to the dependence of $\vec{U}_{n}^{\prime \prime}$ on $M_{n}^{\prime}$ ( $M_{n}^{\prime}$ is a Markov chain). However, in our particular setting we do, because we assume the distribution of the speed vector (thus of $\vec{U}_{n}$ ) is completely symmetric. Indeed, for any non random point $m, J_{m}\left(\vec{U}_{n}\right)$ has the same distribution as $\vec{U}_{n}$. It follows that for any point $M, J_{M}\left(\vec{U}_{n}\right)$ is independent of $M$ and in particular, $J_{M_{n}^{\prime}}\left(\vec{U}_{n}\right)$ is independent of $M_{n}^{\prime}$ and thus $\vec{U}_{n}^{\prime \prime}$ is independent of $\left(M_{k}^{\prime \prime}\right)_{k=0}^{n}$.

By Lemma 2 we can now conclude that $M_{n}^{\prime \prime}$ uniformly converges to the uniform distribution on $[0,2) \times[0,2)$, where "uniformly" is in the sense of Lemma 2. Now for any measurable part $B \subset[0,1) \times[0,1)$ and for any initial value $m \in[0,1) \times[0,1):$

$$
\mathbb{P}_{m}\left(M_{n} \in B\right)=\sum_{i, j \in\{0,1\}} \mathbb{P}_{m}\left(M_{n}^{\prime \prime} \in B_{i, j}^{\prime \prime}\right)
$$

where

$$
B_{i, j}^{\prime \prime}=J_{(i, j)}(B)+(i, j) .
$$

( $J$ is defined in Equation (14), see Figure 12.) The uniform convergence of $M_{n}^{\prime \prime}$ to the uniform distribution, in the sense of Lemma 2, follows immediately. So do the proofs of H2.i and H2.ii, using a similar reasoning as in Lemma 1.

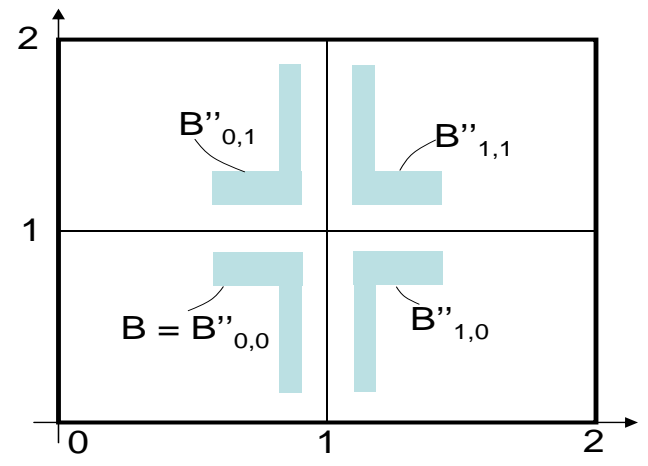

Fig. 12. Mapping from random walk to billiards.

\section{F. Proof of Theorem 6}

Item i. Conditions H1-H3 define the driving chain of phase and path $Y$ to be positive Harris recurrent so that there exists a unique stationary distribution $\pi^{0}$ for the driving chain that is a solution of (1). Combined with H4.i, we have that $\left(S_{n}\right)_{n=0}^{\infty}$ can be defined as a Palm stationary sequence by letting $\pi^{0}$ be the distribution of $Y_{0}$. We now appeal to the conditions of the Slivnyak's inverse construction [4]: (S.i) $0<\mathbb{E}^{0}\left(T_{1}\right)<+\infty$, 
(S.ii) $\mathbb{P}^{0}\left(T_{1}>0\right)=1$, and (S.iii) $\mathbb{E}^{0}(N(0, t])<+\infty$, for all $t \leq t_{0}$ and some $t_{0}>0$. Here $N(0, t]$ is the number of trip transitions that fall in the interval $(0, t]$. S.ii is true by the model (H4.ii), which also implies $\mathbb{E}^{0}\left(T_{1}\right)>0$ in S.i. The rest of S.i is hypothesis of the result. Condition S.iii follows from H4.iii. Indeed,

$$
\begin{aligned}
\mathbb{E}^{0}(N(0, t]) & =\sum_{n=1}^{\infty} \mathbb{P}^{0}\left(T_{n} \leq t\right) \\
& \leq e^{u t} \sum_{n=1}^{\infty} \mathbb{E}^{0}\left(e^{-u T_{n}}\right), \text { any } u>0,
\end{aligned}
$$

where the last inequality is Chernoff inequality. Define the function $m(u, x)=\mathbb{E}^{0}\left(e^{-u T_{1}} \mid Y_{0}=x\right), u>0, x \in I \times \mathcal{P}$. By definition of trip durations (H4.i):

$$
\begin{aligned}
\mathbb{E}^{0}\left(e^{-u T_{n}}\right)= & \int_{I \times \mathcal{P}} \cdots \int_{I \times \mathcal{P}} m(u, x) m\left(u, y_{1}\right) \cdots m\left(u, y_{n-1}\right) \\
& \cdot \pi^{0}(x) P\left(x, d y_{1}\right) P\left(y_{1}, d y_{2}\right) \cdots P\left(y_{n-1}, d y_{n}\right) .
\end{aligned}
$$

Recall that $P(\cdot, \cdot)$ is the transition semigroup of the chain $Y$ as introduced in Equation (1).

Now, from H4.iii, we have that $m(u, y)<1$ for any $u>0$ and $\pi^{0}$ almost any $y$. It thus follows that there exists $\varepsilon>0$ such that $\mathbb{E}^{0}\left(e^{-u T_{n}}\right)<(1-\varepsilon)^{n}$, and in view of $(21)$, this implies S.iii holds.

The existence of a time-stationary distribution $\pi$ follows by the Palm inversion formula [4]:

$$
\mathbb{E}(f(\Phi(0)))=\frac{1}{\mathbb{E}^{0}\left(S_{0}\right)} \mathbb{E}^{0}\left(\int_{0}^{T_{1}} f(\Phi(s)) d s\right),
$$

by taking $f(x)=1_{x \in B}, B \in I \times \mathcal{P} \times \mathbb{R}_{+}^{2}$. Recall that here we admit the convention under which 0 is an arbitrary time. The Palm inversion also implies uniqueness of $\pi$ and the asserted expression.

Item ii-a. In view of a convergence result in [1], it suffices to show that for $\pi^{0}$ almost any $y \in I \times \mathcal{P}$ and any bounded function $g: I \times \mathcal{P} \times[0,+\infty)^{2} \rightarrow \mathbb{R}^{+}$, the following limit holds:

$$
\begin{aligned}
& \lim _{t \rightarrow+\infty} \mathbb{E}_{y}\left(g\left(Y(t), S^{-}(t), S^{+}(t)\right)\right) \\
= & \lambda \int_{y \in I \times \mathcal{P}} \int_{[0, \infty)} \int_{[0, s)} g(y, u, s-u) d u F(y, d s) \pi^{0}(d y),
\end{aligned}
$$

where $1 / \lambda:=\int_{y \in I \times \mathcal{P}} \int_{[0,+\infty)} F(y, d s) \pi^{0}(y)$. Recall that $S(t)=$ $S_{n}, T_{n} \leq t<T_{n+1}$ is the trip duration of the trip on-going at time $t$ and $S^{-}(t)=t-S(t)$ is the time elapsed on the trip at time $t$. The notation $S^{+}(t):=S(t)-S^{-}(t)$ denotes the time until the next trip transition instant as seen at time $t$.

In order to show that the distribution of $\Phi$ converges to the asserted limit, it suffices to show that (23) holds for functions $g(\cdot)$ of the form $g(y, u, v)=1_{y \in A} 1_{u>u_{0}} 1_{v>v_{0}}$, for $u_{0}, v_{0} \geq 0$ and $A$ a product of intervals in $I \times \mathcal{P}$.

It follows from [1, Corollary 1] that the limit (23) holds if both of the two following conditions hold:

(C.i) $f(y, \cdot)$ is continuous almost everywhere, for any $y \in$ $I \times \mathcal{P}$ that does not lie in a set of zero $\pi^{0}$ measure,

(C.ii) $\int_{I \times P} \sum_{n \in \mathbb{Z}} \sup _{n d \leq s<(n+1) d}|f(y, s)| \pi^{0}(y)<+\infty$, for some $d>0$,

where $f(y, s):=\mathbb{E}_{y}\left(g\left(y, s, T_{1}-s\right) 1_{T_{1}>s}\right)$.
First, we check C.i for $g(y, u, v)=1_{y \in A} 1_{u>u_{0}} 1_{v>v_{0}}$. We have

$$
f(y, s)=1_{y \in A} 1_{s>u_{0}} \mathbb{P}_{y}\left(T_{1}>s+v_{0}\right),
$$

which for any fixed $y \in I \times \mathcal{P}$ is almost everywhere continuous with $s$.

Second we check C.ii for $g(\cdot)$ a bounded function. We have that there exists $K<+\infty$ such that $|f(y, s)| \leq K \mathbb{P}_{y}\left(T_{1}>s\right)$. It is readily seen that $K \sum_{n=0}^{\infty} \mathbb{P}_{y}\left(T_{1}>n d\right)$ upper bounds the left-hand side in the inequality C.ii. Further,

$$
\begin{aligned}
\sum_{n=0}^{\infty} \mathbb{P}_{y}\left(T_{1}>n d\right) & \leq \sum_{n=0}^{\infty} \int_{[n d,(n+1) d)} \mathbb{P}_{y}\left(T_{1}>s\right) d s \\
& =\int_{[0,+\infty)} \mathbb{P}_{y}\left(T_{1}>s\right) d s=1 / \lambda .
\end{aligned}
$$

Hence, C.ii is implied by the boundedness of $g(\cdot)$ and finiteness of $1 / \lambda$.

Item ii-b follows from (23).

\section{G. Proof of Theorem 7}

We use the inversion formula of Palm calculus [3]. Let $\lambda$ be the intensity of the point process $T_{n}$, i.e. the average number of trip origins per time unit. For any bounded, non random, function $\phi$ of the process state:

$$
\begin{aligned}
\mathbb{E}(\phi(Y(t), S(t), U(t))) & =\lambda \mathbb{E}^{0}\left(\int_{0}^{S_{0}} \phi\left(Y_{0}, S_{0}, \frac{\tau}{S_{0}}\right) d \tau\right) \\
& \left.=\lambda \mathbb{E}^{0}\left(S_{0} \int_{0}^{1} \phi\left(Y_{0}, S_{0}, u\right) d u\right) 24\right)
\end{aligned}
$$

where the latter is by the change of variable $\tau=S_{0} u$ in the integral. First take $\phi(y, s, u)=1$ and obtain

$$
\lambda=\frac{1}{\int_{Y} \bar{\tau}(y) \pi^{0}(d y)} .
$$

Second, take $\phi(y, s, u)=\psi(y)$ in Equation (24) and obtain

$$
\mathbb{E}(\psi(Y(t)))=\lambda \mathbb{E}^{0}\left(S_{0} \psi\left(Y_{0}\right)\right)=\lambda \int_{Y} \bar{\tau}(y) \pi^{0}(d y)
$$

which shows item 1 . Now take $\phi(y, s)=\psi(y, s)$ and obtain

$$
\begin{aligned}
& \mathbb{E}(\psi(Y(t), S(t)))=\lambda \mathbb{E}^{0}\left(S_{0} \psi\left(Y_{0}, S_{0}\right)\right) \\
& \quad=\lambda \int_{Y} \int_{0}^{+\infty} s \psi(y, s) F(y, d s) \pi^{0}(d y)
\end{aligned}
$$

which shows item 2. Last, take $\phi(y, s, u)=\psi(y, s) \xi(u)$ and obtain

$$
\begin{aligned}
& \mathbb{E}(\psi(Y(t), S(t)) \xi(U(t))) \\
& =\lambda \mathbb{E}^{0}\left(S_{0} \psi\left(Y_{0}, S_{0}\right) \int_{0}^{1} \xi(u) d u\right) \\
& =\lambda\left(\int_{0}^{1} \xi(u) d u\right) \mathbb{E}^{0}\left(S_{0} \psi\left(Y_{0}, S_{0}\right)\right) .
\end{aligned}
$$

This factorization shows that $U(t)$ on one hand, $(Y(t), S(t))$ on the other, are mutually independent ([16, Lemma in Appendix]). Further, let $\psi(\cdot)=1$ and obtain that the distribution of $U(t)$ is uniform on $[0,1]$, which ends the proof of item 3. 


\section{H. Proof of Lemma 7}

Let $I_{k}$ be the phase drawn at the $k$ iteration of the loop and $T$ be the number of iterations when we exit the loop (if ever). Assume first that $\omega_{i}$ is unknown for all $i$. We have $\mathbb{P}(T=k)=q_{1}\left(1-q_{1}\right)^{k-1}$ with

$$
q_{1}=\sum_{i} \int_{\mathbb{R}^{d}} \frac{k_{i}(y)}{\kappa_{i}} g_{i}(y) d y=\sum_{i} v(i) \frac{\omega_{i}}{\kappa_{i}}=\alpha \sum_{i} \mu(i) \omega_{i} .
$$

Note that $0<q_{1} \leq 1$ thus the loop terminates with probability 1. $I_{T}$ is the value of $i$ when we exit the loop and

$$
\begin{aligned}
& \mathbb{P}\left(I_{T}=i\right)=\sum_{k \geq 1} \mathbb{P}\left(I_{T}=i \text { and } T=k \mid T \geq k\right)\left(1-q_{1}\right)^{k-1} \\
& =\sum_{k \geq 1} \mathrm{v}(i) \frac{\omega_{i}}{\kappa_{i}}\left(1-q_{1}\right)^{k-1}=\mathrm{v}(i) \frac{\omega_{i}}{\kappa_{i}} \frac{1}{q_{1}}=\frac{\mu(i) \omega_{i}}{\sum_{j} \mu(j) \omega_{j}}
\end{aligned}
$$

which shows the result in this case. Second, consider some $i$ for which $\omega_{i}$ is known. Let $g_{i}=f_{i} / \omega_{i}, k_{i}(y)=\omega_{i}$ and $\kappa_{i}=\omega_{i}$. When $I=i$ is drawn, it is kept with probability 1 . Thus the case $\omega_{i}$ is known, is a special case of the previous one.

\section{Proof of Theorem 13}

Item 1 follows from the fact that the speed vector is not altered by wrapping. Item 3 directly follows from Theorem 7 and the discussion after it. We now show item 5 . Recall $P(t)(0)$ is the start position of the current path. By Theorem 7, the time stationary joint density of $P(t)(0)=m, \vec{V}(t)=\vec{v}, S(t)=s$, conditional to a move phase is $\frac{s}{\bar{\tau}_{\text {move }}} f_{\vec{V}}^{0}(\vec{v}) f_{\text {move }}^{0}(s) \operatorname{Unif}(m)$, where $\operatorname{Unif}(\cdot)$ is the uniform density on $\mathcal{A}$. Now $X(t)=$ $w\left(M_{n}+U(t) S_{n} \vec{V}_{n}\right), T_{n} \leq t<T_{n+1}$, and $R(t)=(1-U(t)) S(t)$, where $w(\cdot)$ is the wrapping function defined in Part I, Section III-F. Take any three bounded functions $\phi, \psi, \xi$. Now apply Theorem 7:

$$
\begin{aligned}
\mathbb{E}(\phi(X(t)) \psi(\vec{V}(t)) \xi(R(t)) \mid I(t)=\text { move }) \\
=\int_{0}^{1} \int_{\mathbb{R}^{+}} \int_{\mathbb{R}^{2}}\left(\int_{\mathcal{A}} \phi(w(m+u s \vec{v})) \pi^{0}(d m)\right) . \\
\cdot \psi(\vec{v}) \xi(u s) \frac{s}{\bar{\tau}_{\text {move }}} f_{\vec{V}}^{0}(\vec{v}) F_{\text {move }}^{0}(d s) d \vec{v} d u \\
=\int_{0}^{1} \int_{\mathbb{R}^{+}} \int_{\mathbb{R}^{2}}\left(\int_{\mathcal{A}} \phi(w(m+u s \vec{v})) \operatorname{Unif}(d m)\right) . \\
\cdot \psi(\vec{v}) \xi(u s) \frac{s}{\bar{\tau}_{\text {move }}} f_{\vec{V}}^{0}(\vec{v}) F_{\text {move }}^{0}(d s) d \vec{v} \cdot d u
\end{aligned}
$$

The last equality is because by Lemma 1 in Part I, the stationary distribution of trip endpoint, sampled at an arbitrary endpoint, is uniform. Now by Lemma 8

$$
\int_{\mathcal{A}} \phi(w(m+u s \vec{v})) \operatorname{Unif}(d m)=\int_{\mathcal{A}} \phi(m) \operatorname{Unif}(d m)
$$

thus

$$
\begin{aligned}
(25)= & \int_{\mathcal{A}} \phi(m) \operatorname{Unif}(d m) \cdot \\
& \cdot \int_{\mathbb{R}^{2}} \psi(\vec{v}) f_{\vec{V}}^{0}(\vec{v}) d \vec{v} \int_{0}^{1} \int_{0}^{\infty} \frac{s}{\bar{\tau}_{\text {move }}} \xi(u s) F_{\text {move }}^{0}(d s) d u \\
= & \int_{\mathcal{A}} \phi(m) \operatorname{Unif}(d m) \cdot \\
& \cdot \int_{\mathbb{R}^{2}} \psi(\vec{v}) f_{\vec{V}}^{0}(\vec{v}) d \vec{v} \int_{0}^{\infty} \xi(r) F_{\text {move }}^{0}(d r)
\end{aligned}
$$

where the last equality is by the change of variable $(s, u)$ to $(r, s)$. with $r=u s$ This shows item 5 . Item 4 is analog.
This also shows that, conditional to the phase $I(t)$ being either move or pause, the location $X(t)$ is uniformly distributed. Item 2 follows immediately.

\section{J. Proof of Lemma 8}

First we prove the lemma for $d=1$. It is sufficient to consider $a_{1}=1$. We have

$$
X^{\prime}=X+v \quad \bmod 1 .
$$

Since $X^{\prime}$ is limited to the interval $[0,1]$, its distribution is entirely defined by its Fourier coefficients for $n \in \mathbb{Z}: c_{n}^{\prime}=$ $\mathbb{E}\left(e^{2 i \pi n X^{\prime}}\right)$. By Equation (26) $c_{n}^{\prime}=e^{2 i \pi n v} c_{n}$, where $c_{n}$ is the $n$th Fourier coefficient of the distribution of $X$. Now $X$ is uniform over $[0, a]$ thus $c_{n}=0$ for $n \neq 0$ and $c_{0}=1$. It follows that $c_{n}^{\prime}=c_{n}$ for all $n$.

Now back to the general case, we have shown that all coordinates are uniformly distributed. Further, they are independent because $X$ is uniform and $\vec{v}$ is constant.

\section{K. Proof of Theorem 14}

Item 1 follows from Lemma 5, which says that, in order to continue a path from an intermediate point $m$ it is not needed to know the unreflected speed vector, the instant speed is enough. The rest follows from Theorem 7 and Lemma 9, in a similar way as for Theorem 13. More precisely, with the same notation as in the proof of Theorem 13, we have (recall that we defined, in Part I, proof of Lemma 4, for any location $m \in \mathbb{R}^{2}$ the (linear) operator $J_{m}$ as the one that transforms the unreflected speed vector into the true speed vector, when $m$ is the hypothetical location if there would be no reflection):

$$
\begin{aligned}
\mathbb{E} & (\phi(X(t)) \psi(\vec{V}(t)) \xi(R(t)) \mid I(t)=\text { move }) \\
= & \int_{0}^{1} \int_{\mathbb{R}^{+}} h(s, u) \xi(u s) \frac{s}{\bar{\tau}_{\text {move }}} F_{\text {move }}^{0}(d s) d u
\end{aligned}
$$

where

$$
\begin{aligned}
& h(s, u):= \\
& \quad \int_{\mathbb{R}^{2}} \int_{\mathcal{A}} \psi\left(J_{m+s u \vec{v}}(\vec{v})\right) \phi(b(m+u s \vec{v})) f_{\vec{V}}^{0}(\vec{v}) \pi^{0}(d m) d \vec{v} \\
& =\quad \int_{\mathbb{R}^{2}} \int_{\mathcal{A}} \psi\left(J_{m+s u \vec{v}}(\vec{v})\right) \phi(b(m+u s \vec{v})) f_{\vec{v}}^{0}(\vec{v}) \operatorname{Unif}(d m) d \vec{v}
\end{aligned}
$$

where the latter equality is by Part I, Lemma 4. Now we apply Lemma 9 to $h(s, u)$ with $\alpha=s u, M=$ the location of the mobile at beginning of trip (which is uniform under the stationary distribution) and $\vec{W}=$ the unreflected speed vector for this trip. We have, with the notation of the lemma

$$
h(s, u)=\mathbb{E}\left(\phi\left(M^{\prime}\right) \psi\left(\vec{W}^{\prime}\right)\right)
$$

and thus

$$
\begin{aligned}
& h(s, u)=\mathbb{E}(\phi(M) \psi(\vec{W})) \\
& \quad=\int_{\mathbb{R}^{2}} \int_{\mathcal{A}} \psi(\vec{v}) \phi(m) f_{\vec{v}}^{0}(\vec{v}) \operatorname{Unif}(d m) d \vec{v} .
\end{aligned}
$$

Combine this with Equation (27) and obtain the rest of the theorem. 


\section{Proof of Lemma 9}

It is sufficient to consider the case $a_{1}=a_{2}=1$. Since $J_{m}$ is a linear operator, it is also sufficient to consider the case $\alpha=$ 1. The mapping that transforms $(M=(x, y), \vec{W}=(u, v))$ into $\left(M^{\prime}=\left(x^{\prime}, y^{\prime}\right), \vec{W}^{\prime}=\left(u^{\prime}, v^{\prime}\right)\right)$ is such that $x=\varepsilon_{1}\left(x^{\prime}-u^{\prime}\right)+2 n_{1}$, $y=\varepsilon_{2}\left(y^{\prime}-v^{\prime}\right)+2 n_{2}, u=\varepsilon_{1} u^{\prime}$ and $v=\varepsilon_{2} v^{\prime}$, where $\varepsilon_{1}, \varepsilon_{2} \in$ $\{-1,1\}$ and $n_{1}, n_{2} \in \mathbb{Z}$. It is differentiable almost everywhere and its Jacobian is 1 . Thus, the joint density of $\left(M^{\prime}, \vec{W}^{\prime}\right)$ is

$$
\begin{aligned}
& f_{M^{\prime}, \vec{W}^{\prime}}\left(x^{\prime}, y^{\prime}, u^{\prime}, v^{\prime}\right) \\
& =\sum_{\varepsilon_{1}, \varepsilon_{2} \in\{-1,1\}, n_{1}, n_{2} \in \mathbb{Z}} f_{\vec{W}}\left(\varepsilon_{1} u^{\prime}, \varepsilon_{2} v^{\prime}\right) \cdot 1_{\varepsilon_{1}\left(x^{\prime}-u^{\prime}\right)+2 n_{1} \in(0,1)} \\
& \text { - } 1_{\varepsilon_{2}\left(y^{\prime}-v^{\prime}\right)+2 n_{2} \in(0,1)} \text {. }
\end{aligned}
$$

Since $f_{\vec{W}}$ is completely symmetric:

$$
\begin{aligned}
= & f_{\vec{W}}\left(u^{\prime}, v^{\prime}\right) \cdot \\
& \cdot \sum_{\varepsilon_{1}, \varepsilon_{2} \in\{-1,1\}, n_{1}, n_{2} \in \mathbb{Z}} 1_{\varepsilon_{1}\left(x^{\prime}-u^{\prime}\right)+2 n_{1} \in(0,1)} 1_{\varepsilon_{2}\left(y^{\prime}-v^{\prime}\right)+2 n_{2} \in(0,1)} \\
= & \left(\sum_{\varepsilon_{1} \in\{-1,1\}, n_{1} \in \mathbb{Z}} 1_{\varepsilon_{1}\left(x^{\prime}-u^{\prime}\right)+2 n_{1} \in(0,1)}\right) . \\
& \cdot\left(\sum_{\varepsilon_{2} \in\{-1,1\}, n_{2} \in \mathbb{Z}} 1_{\varepsilon_{2}\left(y^{\prime}-v^{\prime}\right)+2 n_{2} \in(0,1)}\right) .
\end{aligned}
$$

Now for any $x \in \mathbb{R} \backslash \mathbb{Z}$ :

$$
\sum_{\varepsilon_{1} \in\{-1,1\}, n_{1} \in \mathbb{Z}} 1_{\left\{\varepsilon_{1} x+2 n_{1} \in(0,1)\right\}}=1 .
$$

It follows that for all $u^{\prime}, v^{\prime}$ and $x^{\prime}, y^{\prime} \in(0,1)$ except on a set of zero mass:

$$
f_{M^{\prime}, \vec{W}^{\prime}}\left(x^{\prime}, y^{\prime}, u^{\prime}, v^{\prime}\right)=f_{\vec{W}}\left(u^{\prime}, v^{\prime}\right)=f_{M, \vec{W}}\left(x^{\prime}, y^{\prime}, u^{\prime}, v^{\prime}\right) .
$$

\section{Evaluation of Transient Path Distribution}

In this section we describe the computation of the transient path distribution in the example in Figure 2. The model is a restricted random waypoint described as follows. The set of subdomains is finite with a subdomain $\mathcal{A}_{i}$ defined as the location of a vertex $i \in \mathcal{L}$. The set of paths is finite and a path is specified by the indexes of the origin and destination vertex $i$ and $j$. To simplify, we consider a model with no pauses. Our objective is to compute the distribution of the path $P(t)$ at time $t \geq 0$ with $t=0$ taken as origin of a trip, i.e. $T_{0}=0$. We are thus interested in $\mathbb{P}^{0}(P(t)=i), t \geq 0, i \in \mathcal{L}$. We can compute this transient distribution as $\left(P_{n}, T_{n}\right)$ by using Markov renewal property as follows: for each $i \in \mathcal{L}$,

$$
\begin{aligned}
& \mathbb{P}^{0}\left(P(t)=j \mid P_{0}=i\right) \\
= & \mathbb{P}^{0}\left(P(t)=j, T_{1}>t \mid P_{0}=i\right)+ \\
& +\sum_{k \in I} \int_{0}^{t} Q(i, k) F_{i}(k, d s) \mathbb{P}^{0}\left(P(t-s)=k \mid P_{0}=k\right)(28)
\end{aligned}
$$

where $F_{i}(j, s):=\mathbb{P}^{0}\left(P_{1}=j, T_{1} \leq s \mid P_{0}=i\right)$. From [11, Proposition 4.9, Chapter 10, Section 4], we have

$$
\begin{aligned}
& \lim _{t \uparrow \infty} \mathbb{P}^{0}\left(P(t)=j \mid P_{0}=i\right) \\
= & \lambda \sum_{k \in \mathcal{L}} q^{0}(k) \int_{0}^{\infty} \mathbb{P}^{0}\left(P(s)=j, T_{1}>s \mid P_{0}=k\right) d s \\
= & \lambda \sum_{k \in \mathcal{L}} q^{0}(k) \mathbb{E}^{0}\left(\int_{0}^{\infty} 1_{P(s)=j} 1_{T_{1}>s} d s \mid P_{0}=k\right) \\
= & \lambda \sum_{k \in \mathcal{L}} q^{0}(k) \mathbb{E}^{0}\left(\int_{0}^{T_{1}} 1_{P(s)=j} d s \mid P_{0}=k\right)
\end{aligned}
$$

where $\lambda=1 / \sum_{k \in \mathcal{L}} q^{0}(k) \mathbb{E}^{0}\left(T_{1} \mid P_{0}=k\right)$. Indeed, the right-hand side in the last equality is precisely what would be given by Palm inversion formula for the time-stationary distribution of path. The above result shows convergence with time to the time-stationary distribution of path, from initial path chosen arbitrarily on the set of paths.

The system of equations (28) is known as Markov renewal equations and can be, in principle, routinely solved numerically. In the example, we assume each path takes a fixed integer number of time units, so for a path $(i, j) \in \mathcal{L}^{2}$, $F_{i}(j, d s)=\delta_{\tau_{j}}(s)$, for some fixed integer $\tau_{j}>0$, where $\delta_{\tau_{j}}(\cdot)$ is a Dirac function. In this case, (28) boils down to the system of difference equations:

$p(i, j, t)=g(i, j, t)+\sum_{k \in \mathcal{L}} Q(i, k) h(k, t) p\left(k, j, t-\tau_{j}\right), t=1,2, \ldots$

where we define $p(i, j, t):=\mathbb{P}^{0}(P(t)=j \mid P(0)=i), g(i, j, t)=$ $1_{\tau_{i}>t, i=j}$ and $h(k, t)=1_{t \geq \tau_{k}}, i, j, k \in \mathcal{L}$. The numerical results in Figure 2 are obtained from the last difference equations.

\section{N. Perfect Sampling for Restricted Random Waypoint}

The theorem generalises Theorem 10 in Section VI, to any number $L$ of sub-domains $\mathcal{A}_{i}, i \in \mathcal{L}$.

Theorem 15: The time stationary distribution $\pi$ of the phase $I(t)=(i, j, r, \phi)$ is

$$
\begin{aligned}
\pi(i, j, r, \phi)= & \lambda q^{0}(i) Q(i, j) \times \\
& \times \begin{cases}\bar{F}_{i}(r-1)\left(\bar{R}_{i}+1\right) \tau_{i, j} & r \geq 0, \phi=\text { pause } \\
\bar{\Delta}_{i, j} \omega_{i, j} & r=0, \phi=\text { move } \\
\bar{F}_{i}(r-1) \bar{R}_{i} \bar{\Delta}_{i, i} \omega_{i, i} & r>0, \phi=\text { move }\end{cases}
\end{aligned}
$$

where $\lambda$ is a normalising constant, defined by the above equation and $\sum_{i} \pi(i)=1, \bar{F}_{i}(r)=1-F_{i}(r)$ and $\bar{R}_{i}=\sum_{r \geq 0} \bar{F}_{i}(r)$ is the expected number of trips within a subdomain $i \in \mathcal{L}$.

Proof. By substitution in the balance equations, we can verify that the event-stationary distribution of the phase $I_{n}$ is given by

$$
\left\{\begin{array}{l}
\pi^{0}(i, j, r, \text { pause })=\pi^{0}(i, j, r, \text { move }) \\
\pi^{0}(i, j, r, \text { move })=\alpha q^{0}(i) Q(i, j) \bar{F}_{i}(r-1), r \geq 0
\end{array}\right.
$$

with $\alpha$ a normalising constant. The rest follows from Theorem 7 .

The perfect sampling algorithm generalising that in Figure 8 to arbitrary number of subdomains is displayed in Figure 13. 
- $\mathcal{L}_{0}=$ is the set of $(i, j)$ for which $\bar{\Delta}_{i, j}$ is known. Define $K_{i, j}=\bar{\Delta}_{i, j}$, for $(i, j) \in \mathcal{L}_{0}$ and otherwise $K_{i, j}=\Delta_{i, j}$.

- The following three distributions are used:

$$
\left\{\begin{array}{l}
E_{p}(i, j)=q^{0}(i) Q(i, j)\left(\bar{R}_{i}+1\right) \tau_{i, j} / e_{p} \\
E_{m, 0}(i, j)=q^{0}(i) Q(i, j) K_{i, j} \omega_{i, j} / e_{m, 0} \\
E_{m, 1}(i, j)=q^{0}(i) Q(i, j) K_{i, i} \bar{R}_{i} \omega_{i, i} / e_{m, 1}
\end{array}\right.
$$

where $e_{p}, e_{m, 0}, e_{m, 1}$ are normalising constants.

$$
\begin{aligned}
& \text { do forever } \\
& \text { Draw } U_{1} \sim U_{e_{p}}(0,1) \\
& \text { if } U_{1} \leq \frac{e_{p}}{e_{p}+e_{m, 0}+e_{m, 1}} / / \text { decide } \phi(t)=\text { pause } \\
& \text { Draw }(i, j) \text { from the distribution } E_{p}(i, j) \\
& \text { Draw } r \in \mathbb{Z}_{+} \text {with probability } \frac{F_{i}(r-1)}{\bar{R}_{i}+1} \\
& I(t)=(i, j, r, \text { pause }) \text {; leave } \\
& \text { else } / / \text { try } \phi(t)=\text { move } \\
& \text { // first sample } i, j \\
& \text { if } U_{1} \leq \frac{e_{m, 0}}{e_{m, 0}+e_{m, 1}} \\
& \text { Draw }(i, j) \text { from the distribution } E_{m, 0}(i, j) ; r=0 \\
& \text { Set } O:=\mathcal{A}_{i}, \mathcal{D}:=\mathcal{A}_{j}, \Delta:=\Delta_{i j} \\
& \text { else } \\
& \text { Draw }(i, j) \text { from the distribution } E_{m, 1}(i, j) \\
& \quad \operatorname{Draw} r \in \mathbb{Z}_{+} \text {with probability } \frac{F_{i}(r-1)}{\bar{R}_{i}+1} \\
& \text { Set } O:=\mathcal{A}_{i}, \mathcal{D}:=\mathcal{A}_{i}, \Delta:=\Delta_{i i} \\
& \text { if }(i, j) \in \mathcal{L}_{0} \\
& I(t)=(i, j, r, \text { move }) \\
& \text { do } \\
& \quad \operatorname{Draw} M_{0} \sim \operatorname{Unif}(O), M_{1} \sim \operatorname{Unif}(\mathcal{D}) \\
& \quad \operatorname{Draw} U_{2} \sim \operatorname{Unif}(0, \Delta) \\
& \text { until } U_{2}<d\left(M_{0}, M_{1}\right) \\
& \text { leave } \\
& \text { else } / /(i, j) \notin \mathcal{L}_{0} \\
& \text { Draw } M_{0} \sim \operatorname{Unif}(O), M_{1} \sim \operatorname{Unif}(\mathcal{D}) \\
& \text { Draw } U_{2} \sim \operatorname{Unif}(0, \Delta) \\
& \text { if } U_{2}<d\left(M_{0}, M_{1}\right) \\
& I(t)=(i, j, r, \text { move }) ; \text { leave } \\
& \text { end do } \\
& \hline
\end{aligned}
$$

\section{end do}

Fig. 13. Sampling algorithm for restricted random waypoint with an arbitrary value of $L$, supporting both cases where the average distance between $\mathcal{A}_{i}$ and $\mathcal{A}_{j}$ is known or not.

\section{O. Details of Perfect Sampling for Restricted Random Way-} point

Complexity. We compare the complexity of the two branches of the algorithm in numbers of calls to the random number generator. Let $a$ be the number of such calls required to simulate one sample $\left(M_{0}, M_{1}\right)$ uniformly in the $\mathcal{A}_{1}$ plus one ( $a=5$ for a rectangle or a disk, usually more for non convex domains). By an analysis similar to the proof of Lemma 7, we find, for the former case $C_{1}=\frac{\alpha+\Delta a}{\alpha+\bar{\Delta}}$ and for the latter $C_{2}=\frac{\Delta-\bar{\Delta}}{\alpha+\bar{\Delta}}(1+a)+\frac{\alpha+(1+a) \Delta}{\alpha+\Delta}$, with $\alpha=\tau_{\text {pause }} / \omega$.

We always have $C_{2}>C_{1}$; thus if $\bar{\Delta}$ is known with little computational cost, it is always preferable to use the former case (" $\bar{\Delta}$ is known"). In contrast, if $\bar{\Delta}$ is not known, there are two options: (i) compute $\bar{\Delta}$ offline by Monte-Carlo simulation and use the former case (" $\bar{\Delta}$ is known"), or (ii) use the latter case (" $\bar{\Delta}$ is not known"). The optimal choice depends on the number $N$ of mobiles that need to be initialised by the sampling procedure ( $N$ includes the number of replications of the simulation). Clearly, since $C_{2}>C_{1}$, as $N$ goes to $\infty$, and since the cost of the Monte Carlo simulation is incurred only once for all simulation runs, there is a breakpoint $N_{0}$ such that for $N \leq N_{0}$ it is optimal to use the first option, and vice versa. The complexity of Monte Carlo to compute $\bar{\Delta}$ with $99.99 \%$ confidence interval and a relative accuracy of $1-\varepsilon$ is of the order of $a\left(6 \frac{\sigma}{\Delta \varepsilon}\right)^{2}$, where $\sigma^{2}$ is the variance of the distance between two points in $\mathcal{A}_{1}$. $\sigma$ depends on the regularity of the domain $\mathcal{A}$. For restricted random waypoint or city graph, it is large compared to the mean value. For more regular areas, a crude approximation of $\sigma$ is $\Delta-\bar{\Delta}$. Comparing $C_{2} / C_{1}$ to this complexity, we find that $N_{0}$ is of the order of 10 to 1000 times $\frac{1}{\varepsilon^{2}}$. In practise, $\varepsilon=10^{-4}$ and thus $N_{0}$ is of the order of $10^{9}$ to $10^{11}$ for $L=1$, which is probably larger than the number of simulation runs performed in a campaign by several orders of magnitude. Thus, it should generally be much more efficient to consider the second option. 